\title{
Implication of Mineralogy and Isotope Data on the Origin of the Permian Basic Volcanic Rocks of the Hronicum (Slovakia, Western Carpathians)
}

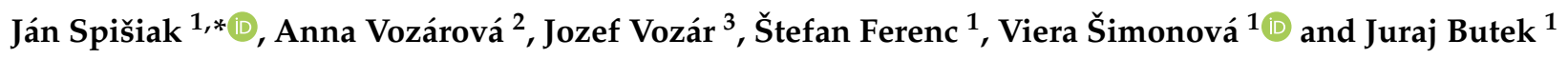 \\ 1 Department of Geography and Geology, Faculty of Natural Sciences, Matej Bel University, Tajovského 40, \\ SK-97401 Banská Bystrica, Slovakia; stefan.ferenc@umb.sk (Š.F.); viera.simonova@umb.sk (V.Š.); \\ juraj.butek@umb.sk (J.B.) \\ 2 Department of Mineralogy and Petrology, Faculty of Natural Sciences, Comenius University, Mlynská Dolina, \\ SK-84215 Bratislava, Slovakia; anna.vozarova@uniba.sk \\ 3 Earth Science Institute Slovak Academy of Science, Dúbravská cesta 9, SK-84005 Bratislava, Slovakia; \\ jozef.vozar@savba.sk \\ * Correspondence: jan.spisiak@umb.sk
}

check for

updates

Citation: Spišiak, J.; Vozárová, A.; Vozár, J.; Ferenc, Š.; Šimonová, V.; Butek, J. Implication of Mineralogy and Isotope Data on the Origin of the Permian Basic Volcanic Rocks of the Hronicum (Slovakia, Western Carpathians). Minerals 2021, 11, 841 https://doi.org/10.3390/min11080841

Academic Editors: Alexey V. Ivanov and Ivan Vlastélic

Received: 25 May 2021

Accepted: 28 July 2021

Published: 4 August 2021

Publisher's Note: MDPI stays neutral with regard to jurisdictional claims in published maps and institutional affiliations.

Copyright: (C) 2021 by the authors Licensee MDPI, Basel, Switzerland. This article is an open access article distributed under the terms and conditions of the Creative Commons Attribution (CC BY) license (https:// creativecommons.org/licenses/by/ $4.0 /)$.

\begin{abstract}
The paper presents new geochemical data of the basic to intermediate volcanic rocks from the Hronic unit. The studied rocks are strongly altered and the primary mineral composition (clinopyroxenes, amphiboles, spinels, biotites, and plagioclases) is preserved only in some localities. The clinopyroxene corresponds to augite and primary amphiboles to pargasites. Spinels have a chemical composition similar to spinels from volcanic rocks. According to chemical composition, the studied basalts correspond to rift-related continental tholeiites. ${ }^{143} \mathrm{Nd} /{ }^{144} \mathrm{Nd}$ vs. ${ }^{87} \mathrm{Sr} /{ }^{86} \mathrm{Sr}$ isotopic ratios of the basalts are scattered around the value of ${ }^{143} \mathrm{Nd} /{ }^{144} \mathrm{Nd}$ for $\mathrm{CHUR}$, where ${ }^{143} \mathrm{Nd} /{ }^{144} \mathrm{Nd}$ ratios are relatively stable and ${ }^{87} \mathrm{Sr} /{ }^{86} \mathrm{Sr}$ values are relatively varied. According to isotopic ratio of ${ }^{207} \mathrm{~Pb} /{ }^{204} \mathrm{~Pb}$ vs. ${ }^{206} \mathrm{~Pb} /{ }^{204} \mathrm{~Pb}$, the basalt analyses lie in the field of EMII (mantle source enriched with crustal materials). The new results of U-Pb LA-ICP-MS dating of apatite point to an age $254 \pm 23$ Ma (Lopingian).
\end{abstract}

Keywords: Western Carpathians; Hronic Unit; Permian volcanic rocks; geochemistry; Sm/Nd and $\mathrm{Pb}$ isotopes

\section{Introduction}

The Pennsylvanian-Permian continental sedimentary basin of the Hronicum represents a type of retroforeland basin [1,2], whose origin has been genetically associated with the convergent regime in the final stages of the Western Carpathian Variscan orogeny. This sedimentary basin is characterized by a huge amount of red-beds type siliciclastic sediments accompanied by basic to intermediate volcanic rocks. Relics of the original filling of this sedimentation area are preserved in today's Western Carpathian structure within a system of superficial nappes, whereas they are completely tectonically detached from their original basement. The crustal character of the original basement was inferred from petrological analysis of sediments [3-5] as well as isotopic ages of clastic mica [6], monazite [7], and zircon [8]. The results documented an active continental margin crust provenance, with dominant Devonian-Mississippian and Pennsylvanian magmatic sources.

This article studied basic to intermediate volcanic rocks whose genesis and chemical composition are closely related to the tectonic processes that led to the formation of the Hronicum Pennsylvanian-Permian sedimentary realm. Previous analyses of the Hronicum basalts/andesites have been focused on their field distribution and description of structural relations and especially on their mineral and chemical composition [9-11]. They were generally classified as rift-related continental tholeiites. 
In this paper, we briefly review the main characteristics of the Permian magmatism in the Hronic unit domain and present new chemical and isotopic data in an attempt to provide some insights into its origin and significance. The main goal of this study is to bring new petrological and geochemical data and identify in more detail the sources of magmas and differentiation processes, and thus infer the relationships with the geotectonic setting of the studied area. Therefore, nine additional samples were collected from the Hronicum Permian volcanic rocks for a better temporal covering of the Hronic formation (Table 1). The studied samples were collected from the Permian volcanites of the 1st and 2nd eruption phases as well as from the dykes crossing Pennsylvanian sediments.

Table 1. Location of samples.

\begin{tabular}{|c|c|c|c|}
\hline $\begin{array}{l}\text { Sample } \\
\text { No. }\end{array}$ & Rock Type & Sample Locality & $\begin{array}{c}\text { GPS } \\
\text { Coordinates }\end{array}$ \\
\hline M-1 & coarse-grained gabbro-diorite dyke & $\begin{array}{l}\text { Malužinská dolina Valley, cliff } \\
\text { on the right slope of the forest } \\
\text { road } 812 \mathrm{~m} \text { a.s.l. }\end{array}$ & $\begin{array}{l}48^{\circ} 57.182^{\prime} \mathrm{N} \\
19^{\circ} 48.783^{\prime} \mathrm{E}\end{array}$ \\
\hline $\mathrm{M}-1 \mathrm{~A}$ & fine-grained gabbro-diorite dyke & $\begin{array}{l}\text { Malužinská dolina Valley, cliff } \\
\text { on the right slope of the forest } \\
\text { road } 812 \mathrm{~m} \text { a.s.l. }\end{array}$ & $\begin{array}{l}48^{\circ} 57.182^{\prime} \mathrm{N} \\
19^{\circ} 48.783^{\prime} \mathrm{E}\end{array}$ \\
\hline M-2 & andesite/basalt-2nd eruption phase & $\begin{array}{l}\text { Hodruša Valley, SE from the } \\
\text { Malužiná Village, cliff on the } \\
\text { right slope of the forest road, } \\
\quad 870 \mathrm{~m} \text { a.s.l. }\end{array}$ & $\begin{array}{l}48^{\circ} 57.552^{\prime} \mathrm{N} \\
19^{\circ} 50.051^{\prime} \mathrm{E}\end{array}$ \\
\hline M-3 & andesite/basalt-2nd eruption phase & $\begin{array}{l}\text { Malužinská dolina Valley, } \\
\text { forest road cut, } 1.4 \text { km SE from } \\
\text { Malužiná Village, } 770 \text { m a.s.l. }\end{array}$ & $\begin{array}{l}48^{\circ} 57.544^{\prime} \mathrm{N} \\
19^{\circ} 50.091^{\prime} \mathrm{E}\end{array}$ \\
\hline M-4 & andesite/basalt-1st eruption phase & $\begin{array}{l}\text { N from the Liptovská Teplička } \\
\text { Village, abandoned quarry } \\
\text { along the Šuňava-Liptovská } \\
\text { Teplička road, } 890 \text { m a.s.l. }\end{array}$ & $\begin{array}{l}48^{\circ} 59.038^{\prime} \mathrm{N} \\
20^{\circ} 05.353^{\prime} \mathrm{E}\end{array}$ \\
\hline M-5 & basaltic andesite-1st eruption phase & $\begin{array}{l}\text { Benkovský potok Valley, small } \\
\text { quarry at the left side of the } \\
\text { forest road, } 832 \mathrm{~m} \text { a.s.l. }\end{array}$ & $\begin{array}{l}49^{\circ} 00.468^{\prime} \mathrm{N} \\
19^{\circ} 59.747^{\prime} \mathrm{E}\end{array}$ \\
\hline M-7 & basaltic andesite-1st eruption phase & $\begin{array}{l}\text { S from the Hranovnica Village, } \\
\text { forest road at the left slope of } \\
\text { the Vernár-Hranovnica state } \\
\text { road, } 694 \mathrm{~m} \text { a.s.l. }\end{array}$ & $\begin{array}{l}48^{\circ} 57.970^{\prime} \mathrm{N} \\
20^{\circ} 17.566^{\prime} \mathrm{E}\end{array}$ \\
\hline M-8 & coarse-grained gabbro-diorite dyke & $\begin{array}{l}\text { West end of the Nižná Boca } \\
\text { Village, } 860 \text { m a.s.l. }\end{array}$ & $\begin{array}{l}48^{\circ} 56.915^{\prime} \mathrm{N} \\
19^{\circ} 46.157^{\prime} \mathrm{E}\end{array}$ \\
\hline M-9 & andesite/basalt-2nd eruption phase & Kvetnica quarry, $725 \mathrm{~m}$ a.s.l. & $\begin{array}{l}49^{\circ} 00.521^{\prime} \mathrm{N} \\
20^{\circ} 17.085^{\prime} \mathrm{E}\end{array}$ \\
\hline
\end{tabular}

\section{Geological Background}

The Hronic nappe system (Figure 1) structurally represents the highest tectonic unit in the Central Western Carpathians. There is no generally accepted opinion about the structure and composition of the Hronic nappe system and its palaeogeographic position. A lot of theories have emerged in the past four decades of research, with current definition of Hronicum as a rootless superficial nappe system [12,13] that mostly comprises a Carboniferous-Permian volcano-sedimentary sequence and Triassic carbonate sediments (with significant similarities of the latter to the Oberostalpine-type). Jurassic-Lower Cretaceous sediments are preserved only locally [14]. 


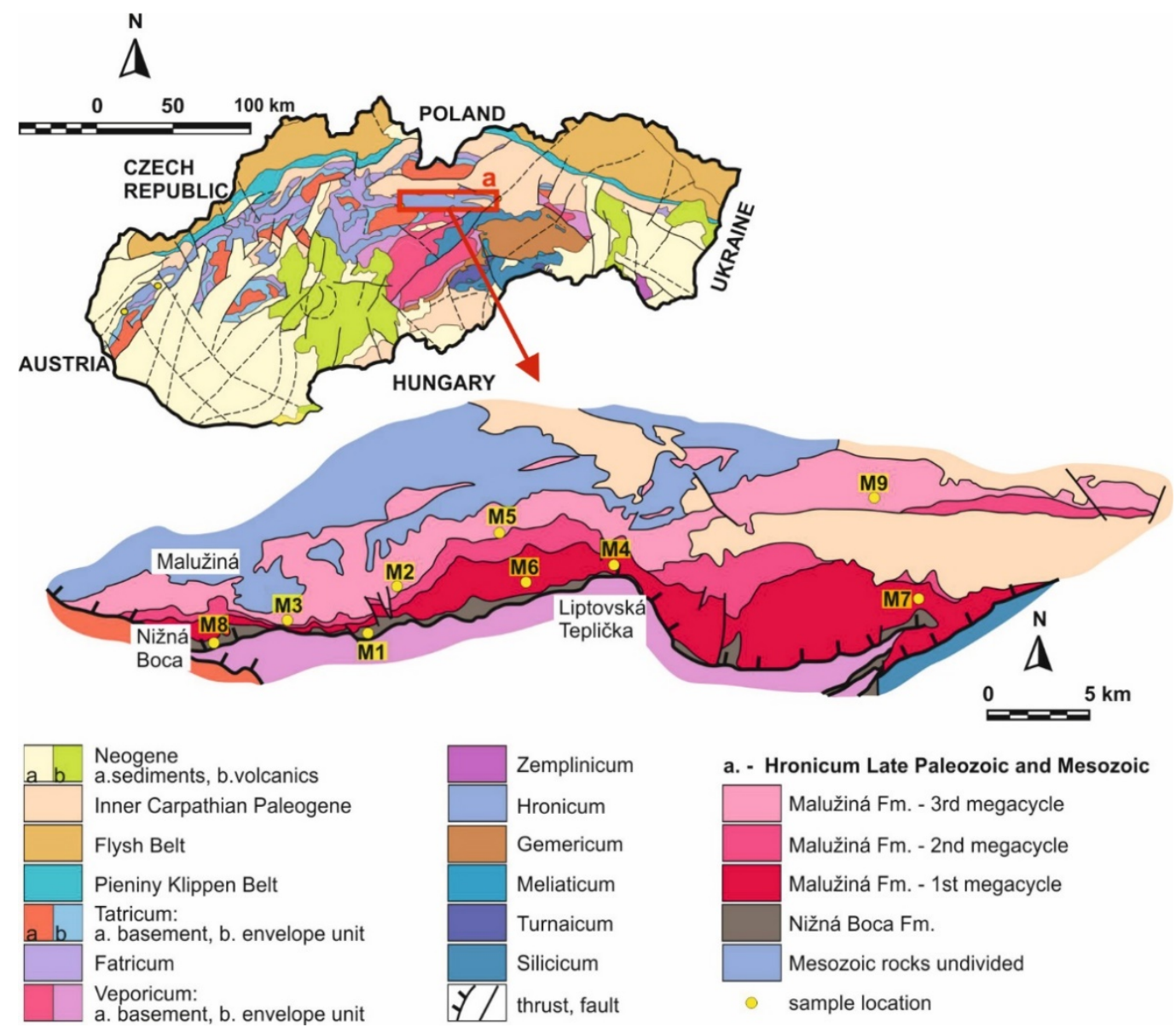

Figure 1. Simplified geological sketch map of the Western Carpathians [15] and schematic geological map of the Hronicum Pennsylvanian-Permian sequence on the northern slopes of the Nízke Tatry Mts. showing sample locations [15].

The allochthonous position of Hronicum superficial nappes relative to basement implies an ultra-Veporic origin. Hronicum probably comes from the area that originally existed between the Veporicum and Gemericum units [16-18]. The southernmost tectonic occurrences of Hronic debris were recognized along the line Lubeník-Margecany (tectonic contact Veporicum/Gemericum). Generally, the Hronicum nappes rest directly on the Fatricum unit, except for Veporicum zone where Hronicum directly overthrust the crystalline complex of Veporicum [19].

The original basement of the Hronicum is currently undetermined. The character of the Pennsylvania-Permian terrestrial siliciclastic sediments suggests that they originated from a sedimentary basin located on the continental crust. This opinion is also supported by findings of some mylonitized granitoid tectonic slices below the basal part of the lower partial Hronicum nappe $[20,21]$. The age of the source area (310-340 Ma) was determined based on the cooling of detrital mica in sandstones (40Ar/39Ar [6]) and suggests that the beginning of the Hronicum sedimentary basin foundation is likely younger than $310 \mathrm{Ma}$ (Moscovian). Analysis of detrital zircon ages indicates the possible derivation of the Hronicum sediments from the Variscan Western Carpathian crystalline basement, mainly the Late Devonian/Early Mississippian magmatic arc (345-370 Ma; [8]). Furthermore, the whole zircon age spectra support close relations between Horonicum and the Armorican terranes and derivation from the Cadomian Belt, which were associated with the West African Craton during the Neoproterozoic and Cambrian time [8], are supported.

The most complete Pennsylvanian-Permian sediments of the Hronic unit have been preserved in the Nízke Tatry Mts (Figure 1). The basal part of the Hronic nappe system is represented by the uppermost Carboniferous-Permian volcano-sedimentary sequence 
called the Ipoltica Group (Figure 1). It comprises the uppermost Carboniferous lowermost Permian Nižná Boca Fm. and the Permian Malužiná Fm. [3,22].

Nižná Boca Formation is the lower lithostratigraphic unit and its age was determined based on the macroflora from the upper part of Nižná Boca Fm as the uppermost Pennsylvanian, Kasimovian-Gzhelian (ICS Stratigraphic Chart 2017). According to the regional stratigraphic scale of Central Europe this correlates with Stephanian [23]. This fact was documented by Sitár and Vozár [24] based on well-preserved macroflora and later by Planderová [25] on the basis of palynology. Nižná Boca Fm. represents a cyclic grey-to-black clastic sequence with a distinct tendency of coarsening upwards. It consists of a multiple vertical alteration of regressive lacustrine-deltaic succession. Their unification in upper part indicates mutual prograding from lacustrine delta to fluvial environment. This sedimentary association was interrupted by synsedimentary subaerial volcanism as manifested by abundant redeposited volcanogenic detrital material mixed with non-volcanic detritus. Thin layers of dacite tuffs are present but are less frequent and similarly exceptional small lava flows can be observed [26]. Sporadic doleritic sills and dykes, occurring in the upper part of the formation, are regarded as co-magmatic with the main Permian volcanism of the younger Malužiná Fm.

The Malužiná formation represents a volcanosedimentary complex, consisting of polycyclic sequences from small to large sedimentary cycles. Deposits can be classified as two main alluvial systems (alluvium of braided rivers and meandering rivers). This sequences likely represent red beds type of sediments, the formation of which consists of brightly colored, mainly red clay and sandy shales, arkose sandstones, siltstones, conglomerates and locally also evaporites, which are assembled into upwardly refining cycles. The characteristic feature of the Malužiná Fm. is the extensive andesite-basalt continental tholeitic volcanism, which was generated during two main eruption phases [9-11]. They belong to the 1st and 3rd megacycle. The 2nd megacycle consists mainly of fluvial-alluvial clastic rocks with fining-upward trend. Small portions of effusive and volcaniclastic rocks occur at the base of the 2nd megacycle.

The Autunian-Saxonian microflora assemblages, described by Planderová [27] and Planderová and Vozárová [28], from the Malužiná Fm. 1st and 2nd megacycles correspond to the Cisuralian-Guadalupian, according to the Global Stratigraphic Scale (ICS, 2017) and the stratigraphic correlations by Izart et al. [29]. This assumption is supported by the $\mathrm{Pb} / \mathrm{U}$ dating from uranium-bearing layers that have been found within the 2nd mega-cycle (263 $\pm 11 \mathrm{Ma}$, [30]). The important magnetostratigraphic data, confirming the position of the Illawarra Reversal Magnetic Horizon, were obtained from the upper part of the 2nd megacycle. Menning [31,32] proved that this reversal magnetic event occurred at the $265 \mathrm{Ma}$ transition. On the basis of magnetostratigraphic results, as well as the Thuringian microflora described by Planderová [27], the 3rd megacycle is considered to be of the Lopingian age.

Commonly, the grade of regional metamorphism did not exceed the very low-grade boundary (according to pumpellyite+prehnite+quartz assemblage-Vrána and Vozár [33]; illite crystallinity indexes from pelites-Plašienka et al. [34] and Šucha and Eberl [35]).

\section{Analytical Methods}

Silicates were studied using electron microprobe JEOL JXA 8530FE at the Earth Sciences Institute in Banská Bystrica under the following conditions: accelerating voltage $15 \mathrm{kV}$, probe current $20 \mathrm{nA}$, beam diameter $3-8 \mu \mathrm{m}$, ZAF correction, counting time $10 \mathrm{~s}$ on peak, $5 \mathrm{~s}$ on background. The used standards, X-ray lines, and detection limit (in ppm) were: $\mathrm{Ca}(\mathrm{K} \alpha, 25)$-diopside, $\mathrm{K}(\mathrm{K} \alpha, 44)$-orthoclase, $\mathrm{P}(\mathrm{K} \alpha, 41)$-apatite, $\mathrm{F}(\mathrm{K} \alpha$, 167)—fluorite, $\mathrm{Na}(\mathrm{K} \alpha, 43)$-albite, $\mathrm{Mg}(\mathrm{K} \alpha, 41)$-diopside, $\mathrm{Al}(\mathrm{K} \alpha, 42)$-albite, $\mathrm{Si}(\mathrm{K} \alpha$, 63) - quartz, $\mathrm{Ba}(\mathrm{L} \alpha, 72)$-barite, $\mathrm{Fe}(\mathrm{K} \alpha, 52)$-hematite, $\mathrm{Cr}(\mathrm{K} \alpha, 113)-\mathrm{Cr} 2 \mathrm{O} 3, \mathrm{Mn}(\mathrm{K} \alpha$, 59)—rhodonite, $\mathrm{Ti}(\mathrm{K} \alpha, 130)$-rutile, $\mathrm{Cl}(\mathrm{K} \alpha, 12)$-tugtupite, $\mathrm{Sr}(\mathrm{L} \alpha, 71)$-celestite.

The chemical composition of the rocks was determined at the ACME Analytical Laboratories (Vancouver, BC, Canada). Total abundances of major element oxides were 
determined by inductively coupled plasma-emission spectrometry (ICP-ES) following lithium metaborate-tetraborate fusion and dilute nitric acid treatment. Loss on ignition (LOI) was calculated from the difference in weight after ignition to $1000^{\circ} \mathrm{C}$. For the total carbon (TOT/C) and sulfur analysis (TOT/S) by LECO analysis, the samples were heated in an induction furnace to $>1650{ }^{\circ} \mathrm{C}$, which caused volatilization of all $\mathrm{C}$ and $\mathrm{S}$ bearing phases. The vapors were carried through an infrared spectrometric cell wherein the concentrations of $\mathrm{C}$ and $\mathrm{S}$ were determined by the absorption of specific wavelengths in the infrared spectra (ORG/C $=$ TOT $/ \mathrm{C}$ minus graphite $\mathrm{C}$ and carbonate). The concentrations of trace elements and rare earth elements were determined by ICP mass spectrometry (ICPMS). Further details are accessible on the web page of the ACME Analytical Laboratories (http:/ / acmelab.com/, accessed on 9 November 2019).

The samples were analyzed for $\mathrm{Pb}$ and $\mathrm{Sr}$ isotopes using a VG Sector 54 IT thermal ionization mass spectrometer (TIMS) at the Department of Geosciences and Natural Resource Management, University of Copenhagen, Denmark. The samples were dissolved in concentrated $\mathrm{HNO}_{3}, \mathrm{HCl}$ and $\mathrm{HF}$ within Savillex ${ }^{\mathrm{TM}}$ beakers on a hotplate at $130{ }^{\circ} \mathrm{C}$ for 3 days [36,37]. Chromatographic separation columns charged with $12 \mathrm{~mL}$ AG50WX 8 (100-200 mesh) cation resin were used for Sr separation. Strontium fractions were purified using a standardized $3 \mathrm{M} \mathrm{HNO}_{3}-\mathrm{H}_{2} \mathrm{O}$ elution recipe on self-made disposable mini-extraction columns including mesh 50-100 SrSpec ${ }^{\mathrm{TM}}$ (Eichrom Inc., Lisle, IL, USA) resin. Separation of $\mathrm{Pb}$ was performed using conventional glass and miniature glass stem anion exchange columns containing $1 \mathrm{~mL}$ and $200 \mu \mathrm{L}$ of 100-200 mesh Bio-Rad AG $1 \times 8$ resin, respectively. Lead isotopes were measured in a static multi-collection mode and fractionation was controlled by repeated analysis of the NBS 981 standard (using values of [38]. Total procedural blanks were below $200 \mathrm{pg} \mathrm{Pb}$, which do not influence lead isotope ratios beyond the significant third digit. Hundred ng loads of the NBS $987 \mathrm{Sr}$ standard gave ${ }^{87} \mathrm{Sr} /{ }^{86} \mathrm{Sr}=0.710238 \pm 0.000018(n=5,2 \sigma)$. The ${ }^{87} \mathrm{Sr} /{ }^{86} \mathrm{Sr}$ values of the samples were corrected for the offset relative to the certified NIST SRM 987 value of 0.710245 [39].

Apatite crystals were separated using standard techniques. Apatite U-Pb data were acquired using a Photon Machines Analyte Exite $193 \mathrm{~nm}$ ArF Excimer laser-ablation system coupled to a Thermo Scientific iCAP Qc at the Department of Geology Trinity College Dublin. Twenty-eight isotopes $\left({ }^{31} \mathrm{P},{ }^{35} \mathrm{Cl},{ }^{43} \mathrm{Ca},{ }^{55} \mathrm{Mn},{ }^{86} \mathrm{Sr},{ }^{89} \mathrm{Y},{ }^{139} \mathrm{La},{ }^{140} \mathrm{Ce},{ }^{141} \mathrm{Pr},{ }^{146} \mathrm{Nd}\right.$, ${ }^{147} \mathrm{Sm},{ }^{153} \mathrm{Eu},{ }^{157} \mathrm{Gd},{ }^{159} \mathrm{~Tb},{ }^{163} \mathrm{Dy},{ }^{165} \mathrm{Ho},{ }^{166} \mathrm{Er},{ }^{169} \mathrm{Tm},{ }^{172} \mathrm{Yb},{ }^{175} \mathrm{Lu},{ }^{200} \mathrm{Hg},{ }^{204} \mathrm{~Pb},{ }^{206} \mathrm{~Pb}$, ${ }^{207} \mathrm{~Pb},{ }^{208} \mathrm{~Pb},{ }^{232} \mathrm{Th},{ }^{238} \mathrm{U}$, and mass $\left({ }^{248} / 232 \mathrm{Th}{ }^{16} \mathrm{O}\right)$ were acquired using a $50 \mu \mathrm{m}$ laser spot, a $4 \mathrm{~Hz}$ laser repetition rate, and a fluence of $3.31 \mathrm{~J} / \mathrm{cm} 2$. A ca. $1 \mathrm{~cm}$ sized crystal of Madagascar apatite, which has yielded a weighted average ID-TIMS concordia age of $473.5 \pm 0.7 \mathrm{Ma}[40,41]$, was used as the primary apatite reference material in this study. McClure Mountain syenite apatite (the rock from which the ${ }^{40} \mathrm{Ar} /{ }^{39} \mathrm{Ar}$ hornblende standard MMhb is derived) was used as a secondary standard. McClure Mountain syenite has moderate but reasonably consistent $U$ and Th contents ( $\sim 23 \mathrm{ppm}$ and $71 \mathrm{ppm}-[42])$ and its thermal history, crystallization age (weighted mean ${ }^{207} \mathrm{~Pb} /{ }^{235} \mathrm{U}$ age of $523.51 \pm 2.09 \mathrm{Ma}$ ), and initial $\mathrm{Pb}$ isotopic composition $\left({ }^{206} \mathrm{~Pb} /{ }^{204} \mathrm{~Pb}=17.54 \pm 0.24 ;{ }^{207} \mathrm{~Pb} /{ }^{204} \mathrm{~Pb}=15.47 \pm 0.04\right)$ are known from high-precision TIMS analyses [43]. Durango apatite was also analyzed in this study as a secondary standard. Durango apatite is a distinctive yellow-green fluorapatite widely used as a mineral standard in apatite fission-track and (U-Th)/He dating and apatite electron micro-probe analyses. It is found as large crystals within an open pit iron mine at Cerro de Mercado, Durango, Mexico. The apatite formed between the eruptions of two major ignimbrites, which have yielded a sanidine-anorthoclase ${ }^{40} \mathrm{Ar}-{ }^{39} \mathrm{Ar}$ age of $31.44 \pm 0.18 \mathrm{Ma}$ [44]. NIST 612 standard glass was used as the apatite trace element concentration reference material. The raw isotope data were reduced using the "VizsualAge" data reduction scheme of Petrus and Kamber [45] within the freeware IOLITE package of Paton et al. [46]. User-defined time intervals are established for the baseline correction procedure to calculate session-wide baseline-corrected values for each isotope. The timeresolved fractionation response of individual standard analyses is then characterized using a user-specified down-hole correction model (such as an exponential curve, a linear fit, or a 
smoothed cubic spline). The data reduction scheme then fits this appropriate sessionwide "model" U-Th-Pb fractionation curve to the time-resolved standard data and the unknowns. Sample-standard bracketing is applied after the correction of down-hole fractionation to account for long-term drift in isotopic or elemental ratios by normalizing all ratios to those of the $\mathrm{U}-\mathrm{Pb}$ reference standards. Common $\mathrm{Pb}$ in the apatite standards was corrected using the ${ }^{207} \mathrm{~Pb}$-based correction method using a modified version of the VizualAge DRS that accounts for the presence of variable common $\mathrm{Pb}$ in the primary standard materials [47]. Over the course of two months of analyses, McClure Mountain apatite $\left({ }^{207} \mathrm{~Pb} /{ }^{235} \mathrm{U}\right.$ TIMS age of $523.51 \pm 1.47 \mathrm{Ma}-[43])$ yielded a U-Pb Tera-Wasserburg concordia lower intercept age of $524.5 \pm 3.7 \mathrm{Ma}$ with an MSWD $=0.72$. The lower intercept was anchored using a ${ }^{207} \mathrm{~Pb} /{ }^{206} \mathrm{~Pb}$ value of 0.88198 derived from apatite ID-TIMS total U-Pb isochron [43].

The structural formulas of the minerals were calculated using standard normalization procedure, i.e., clinopyroxenes on 6 oxygens, feldspars on 8 oxygens, spinels on 4 oxygens, and biotite on equivalent of 22 oxygens. $\mathrm{Fe}^{2+}$ and $\mathrm{Fe}^{3+}$ were calculated based on charge balance. The structural formulas of amphiboles were recalculated on 24 anions using the Amphibole classification Excel spreadsheet v1.9.3 [48].

\section{Petrography and Mineralogy}

The rocks studied are strongly altered and the primary mineral composition is preserved only in some localities. Most of the aphanitic and fine-grained volcanites are strongly chloritized and, in places, carbonated. The coarser-grained varieties of rocks are less altered, in places. Clinopyroxene, plagioclase, and amphibole are locally preserved from the primary minerals. In basaltic andesites and basalts of the 1st and 2 nd eruption phase, the predominant textures are intersertal, pilotaxitic, and hyalopilitic, which are characteristic of fine-grained varieties. In relatively coarse-grained varieties, typical for the first eruption phase, the common textures are trachytic, fine-grained porphyritic, and hyaloophitic. In glassy varieties, volcanic glass is devitrified and rich in Fe-Ti oxides. Amygdaloid and vesicular structures have been commonly identified. In the coarser-grained diorite dykes and sills, the ophitic texture is well preserved.

In fine-grained rocks, pyroxene forms hypidiomorphic phenocrysts, but they are completely altered to a mixture of chlorite and carbonate. Its identification is possible only on the basis of crystal shape within pseudomorphoses. The matrix in basalts and basalt andesites is composed of crystallites of plagioclase, devitrified glass, abundant Fe-Ti oxides, and chlorite pseudomorphoses after small crystals of clinopyroxenes. Clinopyroxene in medium- and coarse-grained rocks forms skeletal individuals and intimately overgrows with plagioclase (Figure 2a,c,e,f) within the ophitic texture. The clinopyroxenes are relatively homogenous in chemical composition, with only rarely observed darker zones in larger grains (Figure 2e) rich in $\mathrm{Mg}$, and/or depleted of Fe. However, this is not typical oscillatory or sector zoning. The chemical composition of clinopyroxenes is characterized by relatively low contents of $\mathrm{TiO}_{2}(0.97-1.56 \mathrm{wt} . \%)$ and alkalis $(0.26-0.34 \mathrm{wt.} \%)$ (Table 2). The Cpx from M-8 sample are slightly different; they have higher $\mathrm{TiO}_{2}$ a $\mathrm{MgO}$, and or lower FeO and $\mathrm{Al}_{2} \mathrm{O}_{3}$ contents. It is probably a reflection of the parent rocks composition (Cr-spinels were also identified in M-8 sample). Based on the classification of pyroxenes (Figure 3), these Cpx correspond to augite. Clinopyroxenes are often lined with younger magmatic amphiboles (Figure 2a,b). Similarly, allotriomorphic grains of ilmenites are observed at the edge of clinopyroxenes. Alternatively, these may represent possible exsolutions of $\mathrm{TiO}_{2}$ from the peripheral parts of the clinopyroxenes. 

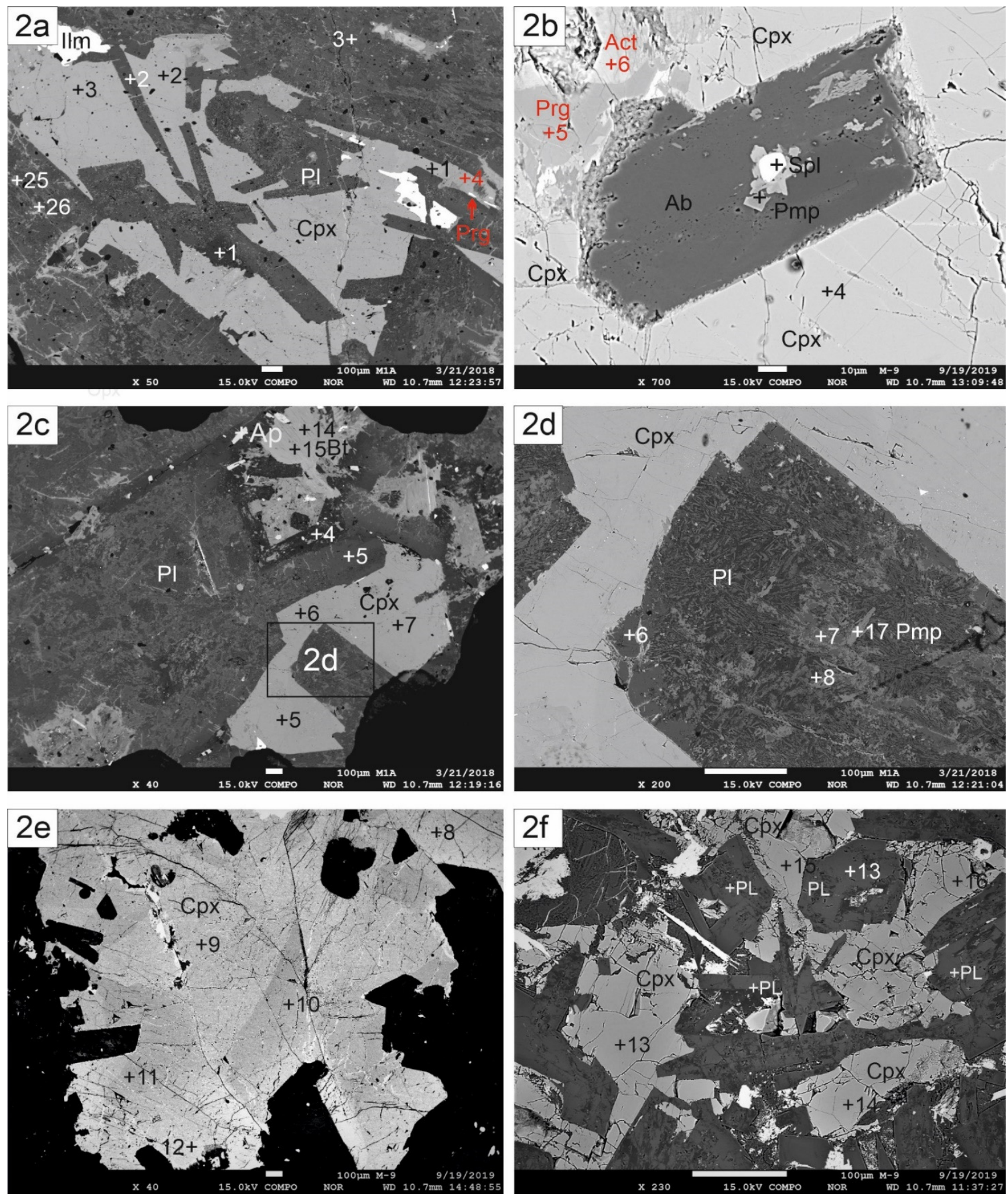

Figure 2. Back scattered electron (BSE) images of basalts from Malužiná; (a)—overgrowth $\mathrm{Pl}$ and $\mathrm{Cpx},(\mathbf{b})$-porphyric Pl around Cpx; (c) -altered Pl, (d)—detail of alterered Pl with Pmp, (e)—zonal porphyric Cpx, (f) -texture of basalt with $\mathrm{Pl}$ and $\mathrm{Cpx} ; \mathrm{Cpx}=$ clinopyroxene, $\mathrm{Prg}=$ pargasite, $\mathrm{Act}=$ actinolite, $\mathrm{Pl}=$ plagioclase, $\mathrm{Bt}=$ biotite, $\mathrm{Pmp}=$ pumpellyite, $\mathrm{Ilm}=$ ilmenite; the numbers in figures correspond to those in Tables 2-5. 
Table 2. Selected analyses of clinopyroxenes.

\begin{tabular}{|c|c|c|c|c|c|c|c|c|c|c|c|c|c|c|c|c|c|c|}
\hline Sample & & M-1 & & M-8 & & M-1 & & \multicolumn{5}{|c|}{ M-8 } & \multicolumn{6}{|c|}{ M-9 } \\
\hline Figures & & $2 a$ & & $2 \mathrm{~b}$ & & $2 c$ & & & & $2 e$ & & & \multicolumn{4}{|c|}{$2 f$} & \multirow[b]{2}{*}{17} & \multirow[b]{2}{*}{18} \\
\hline Anal. $\mathbf{N}$. & 1 & 2 & 3 & 4 & 5 & 6 & 7 & 8 & 9 & 10 & 11 & 12 & 13 & 14 & 15 & 16 & & \\
\hline $\mathrm{SiO}_{2}$ & 51.74 & 50.34 & 50.46 & 52.08 & 50.23 & 50.15 & 50.84 & 50.23 & 50.50 & 51.84 & 51.82 & 51.15 & 50.97 & 50.78 & 50.43 & 50.73 & 50.92 & 50.83 \\
\hline $\mathrm{TiO}_{2}$ & 1.05 & 1.53 & 1.56 & 1.97 & 1.45 & 1.58 & 0.97 & 3.34 & 3.41 & 2.33 & 2.15 & 1.82 & 1.00 & 1.27 & 1.35 & 1.19 & 1.31 & 0.80 \\
\hline $\mathrm{Al}_{2} \mathrm{O}_{3}$ & 1.65 & 2.60 & 2.63 & 0.71 & 2.69 & 2.90 & 2.13 & 1.09 & 0.80 & 0.68 & 0.78 & 0.79 & 2.29 & 2.13 & 2.39 & 2.13 & 2.34 & 2.40 \\
\hline $\mathrm{Cr}_{2} \mathrm{O}_{3}$ & 0.03 & 0.00 & 0.08 & 0.50 & 0.00 & 0.16 & 0.05 & 0.83 & 0.93 & 0.37 & 0.09 & 0.00 & 0.23 & 0.08 & 0.14 & 0.04 & 0.17 & 0.52 \\
\hline $\mathrm{FeO}^{+}$ & 10.87 & 10.21 & 9.91 & 5.90 & 9.68 & 9.31 & 11.23 & 6.03 & 6.04 & 4.57 & 6.61 & 8.95 & 10.65 & 10.43 & 9.63 & 10.57 & 10.03 & 9.28 \\
\hline $\mathrm{MgO}$ & 13.37 & 14.24 & 14.10 & 17.60 & 13.98 & 13.78 & 13.55 & 16.28 & 16.62 & 17.06 & 16.83 & 15.89 & 16.60 & 15.41 & 15.64 & 14.74 & 15.83 & 17.04 \\
\hline $\mathrm{CaO}$ & 19.75 & 19.53 & 19.06 & 20.80 & 19.28 & 19.77 & 19.40 & 21.35 & 21.13 & 21.24 & 21.30 & 20.28 & 17.35 & 19.11 & 19.18 & 19.51 & 18.86 & 17.35 \\
\hline $\mathrm{Na}_{2} \mathrm{O}$ & 0.26 & 0.27 & 0.32 & 0.19 & 0.33 & 0.34 & 0.32 & 0.25 & 0.22 & 0.18 & 0.18 & 0.25 & 0.25 & 0.22 & 0.27 & 0.23 & 0.21 & 0.22 \\
\hline $\mathrm{K}_{2} \mathrm{O}$ & 0.00 & 0.00 & 0.00 & 0.01 & 0.00 & 0.00 & 0.00 & 0.00 & 0.00 & 0.00 & 0.01 & 0.00 & 0.01 & 0.00 & 0.01 & 0.00 & 0.00 & 0.01 \\
\hline Total & 98.96 & 99.02 & 98.28 & 99.88 & 97.93 & 98.22 & 98.73 & 99.51 & 99.84 & 98.36 & 99.88 & 99.38 & 99.57 & 99.63 & 99.22 & 99.29 & 99.75 & 98.69 \\
\hline \multicolumn{19}{|c|}{ Formula based on 6 oxygens } \\
\hline $\mathrm{Si}$ & 1.964 & 1.899 & 1.917 & 1.916 & 1.914 & 1.906 & 1.931 & 1.870 & 1.874 & 1.936 & 1.914 & 1.911 & 1.896 & 1.897 & 1.886 & 1.906 & 1.895 & 1.899 \\
\hline $\mathrm{Ti}$ & 0.030 & 0.043 & 0.045 & 0.054 & 0.042 & 0.045 & 0.028 & 0.094 & 0.095 & 0.065 & 0.060 & 0.051 & 0.028 & 0.036 & 0.038 & 0.034 & 0.037 & 0.023 \\
\hline $\mathrm{Cr}$ & 0.001 & 0.000 & 0.002 & 0.028 & 0.000 & 0.005 & 0.001 & 0.019 & 0.016 & 0.000 & 0.029 & 0.059 & 0.063 & 0.055 & 0.063 & 0.042 & 0.044 & 0.052 \\
\hline $\mathrm{Fe}^{3+}$ & 0.000 & 0.019 & 0.000 & 0.015 & 0.000 & 0.000 & 0.010 & 0.024 & 0.027 & 0.011 & 0.003 & 0.000 & 0.007 & 0.002 & 0.004 & 0.001 & 0.005 & 0.015 \\
\hline $\mathrm{Fe}^{2+}$ & 0.345 & 0.303 & 0.315 & 0.153 & 0.308 & 0.296 & 0.347 & 0.169 & 0.171 & 0.143 & 0.175 & 0.221 & 0.268 & 0.271 & 0.238 & 0.290 & 0.268 & 0.238 \\
\hline $\mathrm{Mn}$ & 0.008 & 0.010 & 0.006 & 0.004 & 0.010 & 0.008 & 0.008 & 0.003 & 0.006 & 0.003 & 0.003 & 0.008 & 0.007 & 0.007 & 0.006 & 0.005 & 0.003 & 0.007 \\
\hline $\mathrm{Mg}$ & 0.757 & 0.801 & 0.798 & 0.965 & 0.794 & 0.781 & 0.767 & 0.903 & 0.919 & 0.950 & 0.927 & 0.885 & 0.921 & 0.858 & 0.872 & 0.826 & 0.879 & 0.949 \\
\hline $\mathrm{Ca}$ & 0.803 & 0.789 & 0.776 & 0.820 & 0.787 & 0.805 & 0.790 & 0.851 & 0.840 & 0.850 & 0.843 & 0.812 & 0.691 & 0.765 & 0.768 & 0.786 & 0.752 & 0.694 \\
\hline $\mathrm{Na}$ & 0.019 & 0.020 & 0.023 & 0.014 & 0.024 & 0.025 & 0.024 & 0.018 & 0.016 & 0.013 & 0.013 & 0.018 & 0.018 & 0.016 & 0.019 & 0.017 & 0.015 & 0.016 \\
\hline $\mathrm{K}$ & 0.000 & 0.000 & 0.000 & 0.000 & 0.000 & 0.000 & 0.000 & 0.000 & 0.000 & 0.000 & 0.000 & 0.000 & 0.000 & 0.000 & 0.000 & 0.000 & 0.000 & 0.000 \\
\hline Wo $\%$ & 42.16 & 41.27 & 41.08 & 42.30 & 41.65 & 42.77 & 41.27 & 44.27 & 43.51 & 43.76 & 43.34 & 42.33 & 36.77 & 40.38 & 40.91 & 41.32 & 39.62 & 36.91 \\
\hline En\% & 39.72 & 41.88 & 42.26 & 49.80 & 42.03 & 41.50 & 40.09 & 46.97 & 47.61 & 48.89 & 47.66 & 46.15 & 48.96 & 45.32 & 46.42 & 43.42 & 46.27 & 50.44 \\
\hline $\mathrm{Fs} \%$ & 18.12 & 16.85 & 16.66 & 7.90 & 16.32 & 15.73 & 18.64 & 8.76 & 8.88 & 7.35 & 9.00 & 11.51 & 14.27 & 14.30 & 12.66 & 15.26 & 14.11 & 12.65 \\
\hline
\end{tabular}


Table 3. Selected analyses of amphiboles.

\begin{tabular}{|c|c|c|c|c|c|c|c|c|c|c|c|c|}
\hline \multirow{2}{*}{$\begin{array}{l}\text { Sample } \\
\text { Anal. N. }\end{array}$} & \multicolumn{4}{|c|}{ M-1 } & \multicolumn{8}{|c|}{ M-8 } \\
\hline & 1 & 2 & 3 & 4 & 5 & 6 & 7 & 8 & 9 & 10 & 11 & 12 \\
\hline Figures & & & & $2 a$ & & & & & & & & \\
\hline $\mathrm{SiO}_{2}$ & 46.77 & 47.14 & 46.23 & 44.03 & 45.73 & 54.07 & 52.98 & 53.19 & 52.62 & 52.75 & 52.05 & 51.92 \\
\hline $\mathrm{TiO}_{2}$ & 0.20 & 0.40 & 0.52 & 2.79 & 0.42 & 0.13 & 0.07 & 0.01 & 0.00 & 0.00 & 0.01 & 0.04 \\
\hline $\mathrm{Al}_{2} \mathrm{O}_{3}$ & 8.55 & 8.43 & 8.89 & 8.86 & 9.85 & 0.61 & 1.58 & 1.74 & 1.44 & 1.52 & 1.68 & 2.05 \\
\hline $\mathrm{Cr}_{2} \mathrm{O}_{3}$ & 0.05 & 0.00 & 0.06 & 0.00 & 0.22 & 0.05 & 0.08 & 0.01 & 0.03 & 0.03 & 0.08 & 0.03 \\
\hline $\mathrm{FeO}^{+}$ & 9.10 & 9.23 & 9.61 & 13.41 & 8.38 & 14.57 & 16.32 & 13.49 & 16.60 & 16.63 & 17.49 & 17.54 \\
\hline $\mathrm{MnO}$ & 0.09 & 0.15 & 0.14 & 0.18 & 0.05 & 0.35 & 0.45 & 0.49 & 0.49 & 0.39 & 0.60 & 0.55 \\
\hline $\mathrm{MgO}$ & 16.91 & 16.47 & 16.51 & 13.27 & 18.33 & 14.66 & 13.84 & 15.82 & 13.33 & 13.34 & 12.35 & 12.14 \\
\hline $\mathrm{CaO}$ & 11.16 & 11.72 & 11.40 & 10.62 & 11.75 & 12.44 & 11.80 & 11.59 & 12.00 & 12.25 & 12.13 & 11.97 \\
\hline $\mathrm{Na}_{2} \mathrm{O}$ & 2.06 & 1.95 & 2.13 & 2.28 & 2.94 & 0.11 & 0.22 & 0.36 & 0.16 & 0.15 & 0.23 & 0.32 \\
\hline $\mathrm{K}_{2} \mathrm{O}$ & 0.42 & 0.34 & 0.45 & 0.53 & 0.17 & 0.04 & 0.04 & 0.02 & 0.04 & 0.04 & 0.06 & 0.05 \\
\hline Total & 95.29 & 95.83 & 95.93 & 95.96 & 97.83 & 97.02 & 97.37 & 96.71 & 96.70 & 97.12 & 96.69 & 96.59 \\
\hline \multicolumn{13}{|c|}{ Formula based on $24(\mathrm{O}, \mathrm{OH}, \mathrm{F}, \mathrm{Cl}$, ) } \\
\hline $\mathrm{Si}$ & 6.824 & 6.867 & 6.738 & 6.588 & 6.496 & 7.898 & 7.756 & 7.724 & 7.776 & 7.759 & 7.740 & 7.731 \\
\hline AlIV & 1.176 & 1.133 & 1.262 & 1.412 & 1.504 & 0.102 & 0.244 & 0.276 & 0.224 & 0.241 & 0.260 & 0.269 \\
\hline $\mathrm{T}$ & 8.000 & 8.000 & 8.000 & 8.000 & 8.000 & 8.000 & 8.000 & 8.000 & 8.000 & 8.000 & 8.000 & 8.000 \\
\hline $\mathrm{Ti}$ & 0.022 & 0.043 & 0.057 & 0.314 & 0.045 & 0.014 & 0.007 & 0.001 & 0.000 & 0.000 & 0.001 & 0.005 \\
\hline AlVI & 0.294 & 0.315 & 0.265 & 0.150 & 0.145 & 0.003 & 0.028 & 0.021 & 0.026 & 0.023 & 0.035 & 0.090 \\
\hline $\mathrm{Cr}$ & 0.006 & 0.000 & 0.006 & 0.000 & 0.025 & 0.006 & 0.010 & 0.002 & 0.003 & 0.004 & 0.010 & 0.003 \\
\hline $\mathrm{Fe}^{3+}$ & 0.445 & 0.300 & 0.425 & 0.217 & 0.631 & 0.056 & 0.184 & 0.247 & 0.188 & 0.206 & 0.202 & 0.156 \\
\hline $\mathrm{Mn}^{2+}$ & 0.000 & 0.000 & 0.000 & 0.000 & 0.000 & 0.006 & 0.000 & 0.000 & 0.000 & 0.002 & 0.042 & 0.024 \\
\hline $\mathrm{Fe}^{2+}$ & 0.556 & 0.764 & 0.661 & 1.360 & 0.272 & 1.723 & 1.752 & 1.305 & 1.847 & 1.839 & 1.973 & 2.027 \\
\hline $\mathrm{Mg}$ & 3.677 & 3.578 & 3.586 & 2.959 & 3.882 & 3.192 & 3.020 & 3.424 & 2.936 & 2.926 & 2.737 & 2.694 \\
\hline$C$ & 5.000 & 5.000 & 5.000 & 5.000 & 5.000 & 5.000 & 5.001 & 5.000 & 5.000 & 5.000 & 5.000 & 4.999 \\
\hline $\mathrm{Mn}^{2+}$ & 0.011 & 0.019 & 0.017 & 0.023 & 0.006 & 0.037 & 0.056 & 0.061 & 0.061 & 0.047 & 0.034 & 0.045 \\
\hline $\mathrm{Fe}^{2+}$ & 0.109 & 0.061 & 0.086 & 0.101 & 0.093 & 0.000 & 0.063 & 0.086 & 0.017 & 0.000 & 0.000 & 0.000 \\
\hline $\mathrm{Ca}$ & 1.745 & 1.829 & 1.781 & 1.702 & 1.788 & 1.947 & 1.851 & 1.803 & 1.900 & 1.931 & 1.933 & 1.909 \\
\hline $\mathrm{Na}$ & 0.136 & 0.091 & 0.117 & 0.173 & 0.113 & 0.016 & 0.031 & 0.051 & 0.023 & 0.022 & 0.033 & 0.046 \\
\hline B & 2.001 & 2.000 & 2.001 & 1.999 & 2.000 & 2.000 & 2.001 & 2.001 & 2.001 & 2.000 & 2.000 & 2.000 \\
\hline $\mathrm{Na}$ & 0.446 & 0.459 & 0.485 & 0.488 & 0.696 & 0.016 & 0.031 & 0.051 & 0.023 & 0.022 & 0.034 & 0.046 \\
\hline $\mathrm{K}$ & 0.077 & 0.063 & 0.084 & 0.101 & 0.031 & 0.008 & 0.008 & 0.004 & 0.007 & 0.008 & 0.012 & 0.009 \\
\hline A & 0.523 & 0.522 & 0.569 & 0.589 & 0.727 & 0.024 & 0.039 & 0.055 & 0.030 & 0.030 & 0.046 & 0.055 \\
\hline
\end{tabular}


Table 4. Selected analyses of biotites, prehnites, and pumpellyites.

\begin{tabular}{|c|c|c|c|c|c|c|c|c|c|}
\hline \multirow{3}{*}{$\begin{array}{c}\text { Sample } \\
\text { Mineral } \\
\text { Anal. N. }\end{array}$} & \multirow{2}{*}{\multicolumn{2}{|c|}{$\begin{array}{c}\text { M1 } \\
\text { Biotite }\end{array}$}} & \multicolumn{3}{|c|}{ M-1 } & \multicolumn{4}{|c|}{ M-8 } \\
\hline & & & \multicolumn{5}{|c|}{ Pumpellyite } & \multicolumn{2}{|c|}{ Prehnite } \\
\hline & 14 & 15 & 17 & 25 & 26 & 2 & 22 & 24 & 27 \\
\hline $\mathrm{SiO}_{2}$ & 37.69 & 37.96 & 36.84 & 37.26 & 37.33 & 37.95 & 37.29 & 43.56 & 43.45 \\
\hline $\mathrm{TiO}_{2}$ & 4.58 & 4.48 & 0.02 & 0.16 & 0.06 & 0.13 & 0.02 & 0.10 & 0.18 \\
\hline $\mathrm{Al}_{2} \mathrm{O}_{3}$ & 12.18 & 12.15 & 25.90 & 25.37 & 25.12 & 29.85 & 26.89 & 24.52 & 24.51 \\
\hline $\mathrm{Cr}_{2} \mathrm{O}_{3}$ & 0.00 & 0.00 & & & & & & & \\
\hline $\mathrm{FeO}^{+}$ & 16.55 & 17.19 & 3.07 & 3.33 & 3.08 & 0.71 & 2.47 & 0.27 & 0.15 \\
\hline $\mathrm{MnO}$ & 0.04 & 0.00 & 0.14 & 0.08 & 0.00 & 0.04 & 0.15 & 0.00 & 0.00 \\
\hline $\mathrm{MgO}$ & 13.40 & 13.11 & 1.71 & 2.29 & 2.28 & 0.09 & 1.98 & 0.00 & 0.03 \\
\hline $\mathrm{CaO}$ & 0.00 & 0.03 & 22.10 & 22.30 & 22.36 & 23.45 & 23.31 & 27.36 & 27.27 \\
\hline $\mathrm{BaO}$ & 0.17 & 1.43 & 0.01 & 0.00 & 0.00 & 0.00 & 0.00 & 0.00 & 0.00 \\
\hline $\mathrm{SrO}$ & 0.00 & 0.00 & 0.02 & 0.01 & 0.00 & 0.00 & 0.00 & 0.00 & 0.00 \\
\hline $\mathrm{Na}_{2} \mathrm{O}$ & 0.14 & 0.17 & 0.13 & 0.04 & 0.01 & 0.03 & 0.03 & 0.06 & 0.02 \\
\hline $\mathrm{K}_{2} \mathrm{O}$ & 9.05 & 9.06 & 0.02 & 0.00 & 0.00 & 0.00 & 0.04 & 0.02 & 0.02 \\
\hline $\mathrm{Cl}$ & 0.09 & 0.11 & & & & & & & \\
\hline $\mathrm{F}$ & 0.19 & 0.00 & & & & & & & \\
\hline Total & 94.07 & 95.67 & 89.94 & 90.83 & 90.23 & 92.26 & 92.18 & 95.88 & 95.63 \\
\hline \multicolumn{3}{|c|}{ Formula based on $24(\mathrm{O}, \mathrm{OH}, \mathrm{Cl}, \mathrm{F})$} & \multicolumn{5}{|c|}{ Formula based on 16 cations and 24,5 oxygens } & \multicolumn{2}{|c|}{ Formula based on $22 \mathrm{O}$ * } \\
\hline $\mathrm{Si}$ & 5.773 & 5.776 & 6.076 & 6.093 & 6.135 & 6.007 & 5.997 & 5.977 & 5.975 \\
\hline $\mathrm{Al} \mathrm{IV}$ & 2.199 & 2.179 & 5.035 & 4.891 & 4.866 & 5.568 & 5.098 & 3.965 & 3.972 \\
\hline $\mathrm{Al} \mathrm{VI}$ & 0.000 & 0.000 & & & & & & & \\
\hline $\mathrm{Ti}$ & 0.528 & 0.512 & 0.003 & 0.019 & 0.007 & 0.016 & 0.003 & 0.011 * & 0.019 * \\
\hline $\mathrm{Cr}$ & 0.000 & 0.000 & & & & & & & \\
\hline $\mathrm{Fe}$ & 2.121 & 2.188 & 0.423 & 0.455 & 0.423 & 0.095 & 0.332 & 0.031 & 0.017 \\
\hline $\mathrm{Mn}$ & 0.005 & 0.000 & 0.019 & 0.010 & 0.000 & 0.005 & 0.020 & 0.000 & 0.000 \\
\hline $\mathrm{Mg}$ & 3.060 & 2.974 & 0.420 & 0.559 & 0.557 & 0.021 & 0.475 & 0.000 & 0.005 \\
\hline $\mathrm{Ca}$ & 0.000 & 0.004 & 3.906 & 3.908 & 3.937 & 3.976 & 4.018 & 4.022 & 4.019 \\
\hline $\mathrm{Na}$ & 0.042 & 0.049 & 0.041 & 0.013 & 0.003 & 0.008 & 0.009 & 0.016 & 0.006 \\
\hline $\mathrm{K}$ & 1.768 & 1.758 & 0.003 & 0.000 & 0.000 & 0.001 & 0.009 & 0.003 & 0.004 \\
\hline $\mathrm{Sr}$ & 0.000 & 0.000 & & & & & & & \\
\hline $\mathrm{Ba}$ & 0.010 & 0.085 & & & & & & & \\
\hline $\mathrm{OH}^{*}$ & 3.884 & 3.972 & & & & & & & \\
\hline $\mathrm{F}$ & 0.092 & 0.000 & & & & & & & \\
\hline $\mathrm{Cl}$ & 0.024 & 0.028 & & & & & & & \\
\hline TOTAL & 19.505 & 19.525 & & & & & & & \\
\hline
\end{tabular}


Table 5. Selected analyses of plagioclases.

\begin{tabular}{|c|c|c|c|c|c|c|c|c|c|c|c|c|c|c|c|}
\hline \multirow{2}{*}{$\begin{array}{c}\text { Sample } \\
\text { Anal. N. }\end{array}$} & \multicolumn{8}{|c|}{ M1A } & \multicolumn{7}{|c|}{ M-9 } \\
\hline & 1 & 2 & 3 & 4 & 5 & 6 & 7 & 8 & 9 & 10 & 11 & 12 & 13 & 14 & 15 \\
\hline & 22 & 23 & 24 & 20 & 21 & 16 & 18 & 19 & 1 & 3 & 4 & 5 & 6 & 7 & 12 \\
\hline Figures & & $2 a$ & & \multicolumn{2}{|c|}{$2 c$} & \multicolumn{3}{|c|}{$2 d$} & & & & & & & \\
\hline $\mathrm{SiO}_{2}$ & 68.92 & 56.18 & 55.06 & 68.67 & 57.82 & 56.34 & 54.01 & 64.48 & 55.14 & 55.27 & 55.78 & 55.27 & 56.26 & 61.22 & 57.38 \\
\hline $\mathrm{TiO}_{2}$ & 0.00 & 0.20 & 0.13 & 0.03 & 0.19 & 0.07 & 0.19 & 0.06 & 0.24 & 0.11 & 0.17 & 0.19 & 0.17 & 0.07 & 0.24 \\
\hline $\mathrm{Al}_{2} \mathrm{O}_{3}$ & 19.21 & 26.84 & 27.75 & 19.32 & 25.81 & 26.66 & 28.35 & 21.62 & 27.75 & 28.12 & 27.73 & 27.86 & 27.33 & 24.18 & 26.45 \\
\hline $\mathrm{FeO}^{+}$ & 0.10 & 0.43 & 0.21 & 0.11 & 0.19 & 0.39 & 0.35 & 0.00 & 0.50 & 0.43 & 0.45 & 0.49 & 0.55 & 0.57 & 0.50 \\
\hline $\mathrm{CaO}$ & 0.10 & 9.34 & 10.34 & 0.09 & 8.12 & 9.40 & 10.99 & 2.80 & 10.57 & 11.04 & 10.71 & 10.58 & 10.19 & 6.02 & 9.34 \\
\hline $\mathrm{BaO}$ & 0.01 & 0.00 & 0.03 & 0.01 & 0.03 & 0.06 & 0.00 & 0.00 & 0.00 & 0.02 & 0.00 & 0.01 & 0.03 & 0.02 & 0.00 \\
\hline $\mathrm{Na}_{2} \mathrm{O}$ & 11.41 & 5.79 & 5.39 & 11.64 & 6.53 & 5.84 & 5.03 & 10.08 & 5.31 & 5.12 & 5.51 & 5.37 & 5.68 & 7.63 & 6.07 \\
\hline $\mathrm{K}_{2} \mathrm{O}$ & 0.09 & 0.18 & 0.21 & 0.05 & 0.37 & 0.29 & 0.23 & 0.09 & 0.25 & 0.21 & 0.24 & 0.25 & 0.23 & 0.62 & 0.30 \\
\hline Total & 99.83 & 98.96 & 99.12 & 99.92 & 99.06 & 99.04 & 99.14 & 99.12 & 99.77 & 100.32 & 100.58 & 100.01 & 100.44 & 100.32 & 100.27 \\
\hline \multicolumn{16}{|c|}{ Formula based on 5 cations and 8 oxygens } \\
\hline $\mathrm{Si}$ & 3.022 & 2.556 & 2.506 & 3.004 & 2.619 & 2.562 & 2.461 & 2.862 & 2.496 & 2.490 & 2.500 & 2.494 & 2.523 & 2.725 & 2.574 \\
\hline $\mathrm{Ti}$ & 0.000 & 0.007 & 0.005 & 0.001 & 0.006 & 0.002 & 0.006 & 0.002 & 0.008 & 0.004 & 0.006 & 0.006 & 0.006 & 0.002 & 0.008 \\
\hline $\mathrm{Al}$ & 0.993 & 1.439 & 1.489 & 0.996 & 1.378 & 1.429 & 1.522 & 1.131 & 1.480 & 1.493 & 1.465 & 1.482 & 1.445 & 1.268 & 1.398 \\
\hline $\mathrm{Cr}$ & 0.000 & 0.000 & 0.000 & 0.000 & 0.000 & 0.000 & 0.000 & 0.000 & 0.000 & 0.000 & 0.000 & 0.000 & 0.000 & 0.000 & 0.000 \\
\hline $\mathrm{Fe}^{3+}$ & 0.000 & 0.000 & 0.000 & 0.000 & 0.000 & 0.000 & 0.000 & 0.000 & 0.000 & 0.000 & 0.000 & 0.000 & 0.000 & 0.000 & 0.000 \\
\hline $\mathrm{Fe}^{2+}$ & 0.004 & 0.016 & 0.008 & 0.004 & 0.007 & 0.015 & 0.013 & 0.000 & 0.019 & 0.016 & 0.017 & 0.019 & 0.021 & 0.021 & 0.019 \\
\hline $\mathrm{Ca}$ & 0.004 & 0.455 & 0.504 & 0.004 & 0.394 & 0.458 & 0.537 & 0.133 & 0.512 & 0.533 & 0.514 & 0.511 & 0.490 & 0.287 & 0.449 \\
\hline $\mathrm{Ba}$ & 0.000 & 0.000 & 0.000 & 0.000 & 0.001 & 0.001 & 0.000 & 0.000 & 0.000 & 0.000 & 0.000 & 0.000 & 0.000 & 0.000 & 0.000 \\
\hline $\mathrm{Na}$ & 0.970 & 0.510 & 0.476 & 0.987 & 0.573 & 0.515 & 0.444 & 0.867 & 0.466 & 0.447 & 0.479 & 0.470 & 0.494 & 0.659 & 0.528 \\
\hline $\mathrm{K}$ & 0.005 & 0.010 & 0.012 & 0.003 & 0.021 & 0.017 & 0.013 & 0.005 & 0.015 & 0.012 & 0.014 & 0.014 & 0.013 & 0.035 & 0.017 \\
\hline An & 0.46 & 46.65 & 50.84 & 0.43 & 39.84 & 46.27 & 53.96 & 13.24 & 51.59 & 53.74 & 51.09 & 51.38 & 49.14 & 29.27 & 45.17 \\
\hline $\mathrm{Ab}$ & 99.05 & 52.28 & 47.93 & 99.27 & 57.99 & 52.03 & 44.70 & 86.25 & 46.94 & 45.05 & 47.56 & 47.19 & 49.54 & 67.17 & 53.13 \\
\hline Or & 0.49 & 1.07 & 1.22 & 0.29 & 2.17 & 1.69 & 1.34 & 0.51 & 1.47 & 1.21 & 1.35 & 1.43 & 1.32 & 3.57 & 1.70 \\
\hline
\end{tabular}

$\mathrm{FeO}^{+}$total $\mathrm{Fe}$ as $\mathrm{FeO}$.

Among other mafic minerals, there occur amphiboles and biotites. Both these minerals are strongly altered. There are two types of amphiboles in the rock (Table 3). It is likely that the older amphibole coexisted with Cpx and has a composition corresponding to pargasite (Figure 4). Pargasite also locally forms rims around Cpx (Figure 2a). The younger amphibole has a composition corresponding to actinolite/tremolite (Figure 4). Compared to the older amphiboles, the younger ones have higher contents of $\mathrm{Si}, \mathrm{Fe}$, or lower $\mathrm{Mg}, \mathrm{Al}$, and $\mathrm{Na}$. 


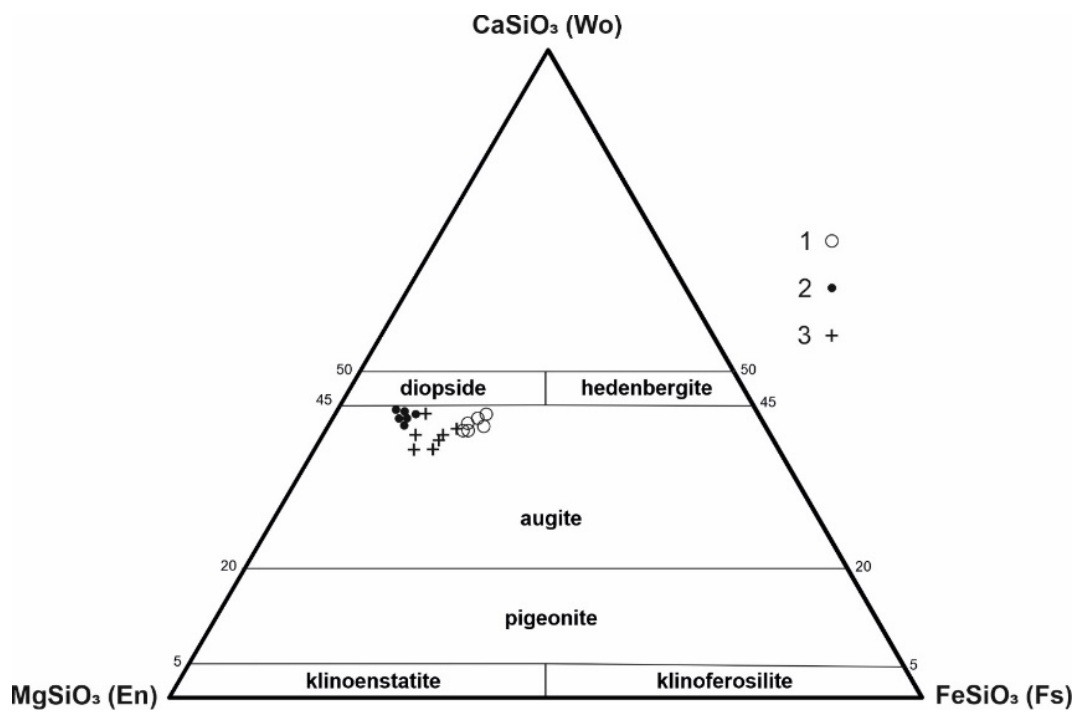

Figure 3. Classification diagram of clinopyroxenes [49]. 1—sample M-1; 2—sample M-8; 3—sample M-9.

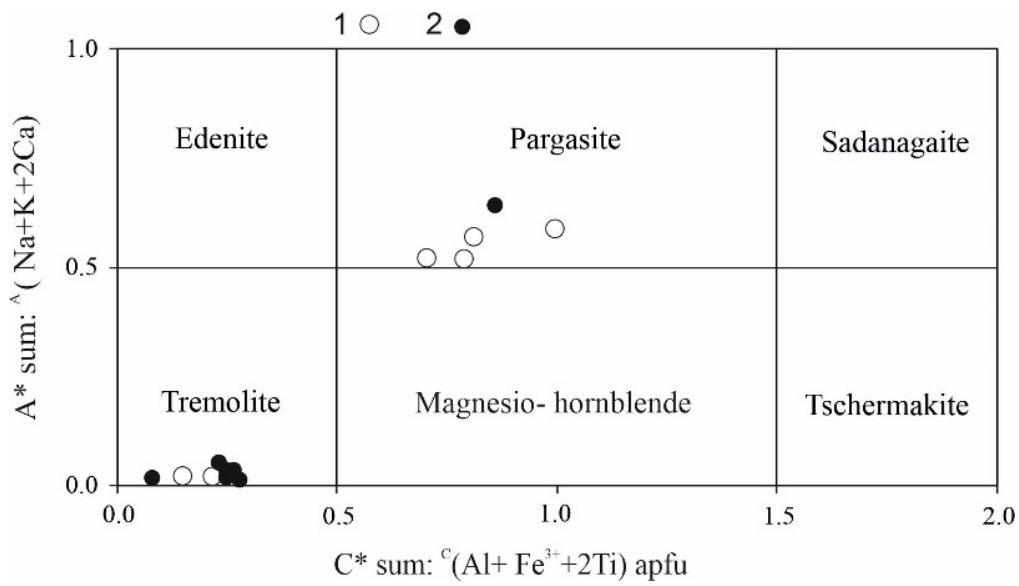

Figure 4. The classification diagram of amphiboles, according Hawthorne et al., [50]. 1—sample M-1; 2-sample M-8.

Biotite is strongly altered and pushes amphibole locally at the edges. It is difficult to determine whether it is primary (Table 4), but the high $\mathrm{TiO}_{2}$ suggests it is magmatic biotite. Based on the classification of Rieder et al. [51] (Figure 5A), the biotite has a high content of annite component. The biotite that makes rims around primary amphibole has increased $\mathrm{MgO}$ content and thus increased content of phlogopite component (Table 4). Abdel-Rahman [52] used a change in the chemical composition of biotites from different genetic basalt types (Figure 5B). The biotites studied lie in the field of calc-alkaline rocks, which correlates well with the overall chemical composition of the rocks studied. 


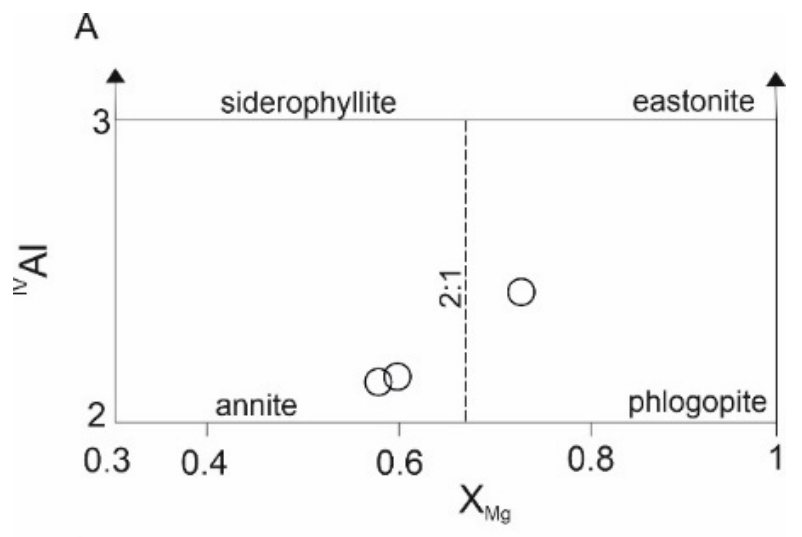

B

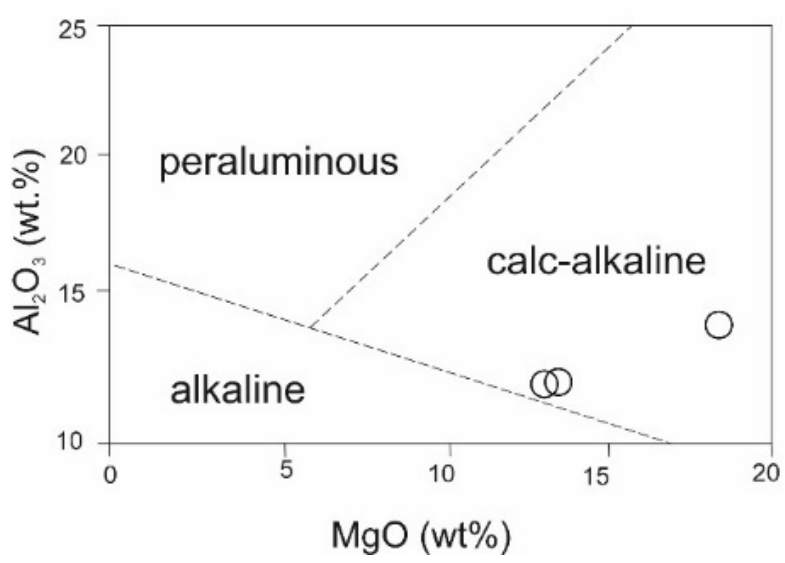

Figure 5. (A) Classification diagram of studied micas. End-members names according to Rieder et al. [51]; (B) Position of studied mica in the classification diagram of Abdel-Rahman [52] for micas from different magmatic series. 1-sample M-1.

Plagioclases in fine-grained strongly altered types, often completely converted to albite, respectively, locally observe tin strips of pumpellyite in the central part of plagioclase (Figure $2 \mathrm{a}, \mathrm{d}$ ). In medium-grained varieties, they are relatively better preserved and relics of primary plagioclases intimately overgrowing with clinopyroxenes can be identified. Overall, in a detailed study, a relatively high inhomogeneity of plagioclases due to postmagmatic and metamorphic processes (Figure 2a,c,d) can be observed. The basicity in the relics of the most preserved primary plagioclases ranges from $40 \%$ to $54 \%$ of the anorthite component, and thus corresponds to andesine to labradorite (Figure 6, Table 5). In the samples with preserved fresh plagioclase, zoning with anorthite component decreasing toward the plagioclase edge can be observed (Core $\mathrm{An}_{49}$, rims $\left.\mathrm{An}_{30}\right)$. The composition of altered parts of plagioclase corresponds to albite. 


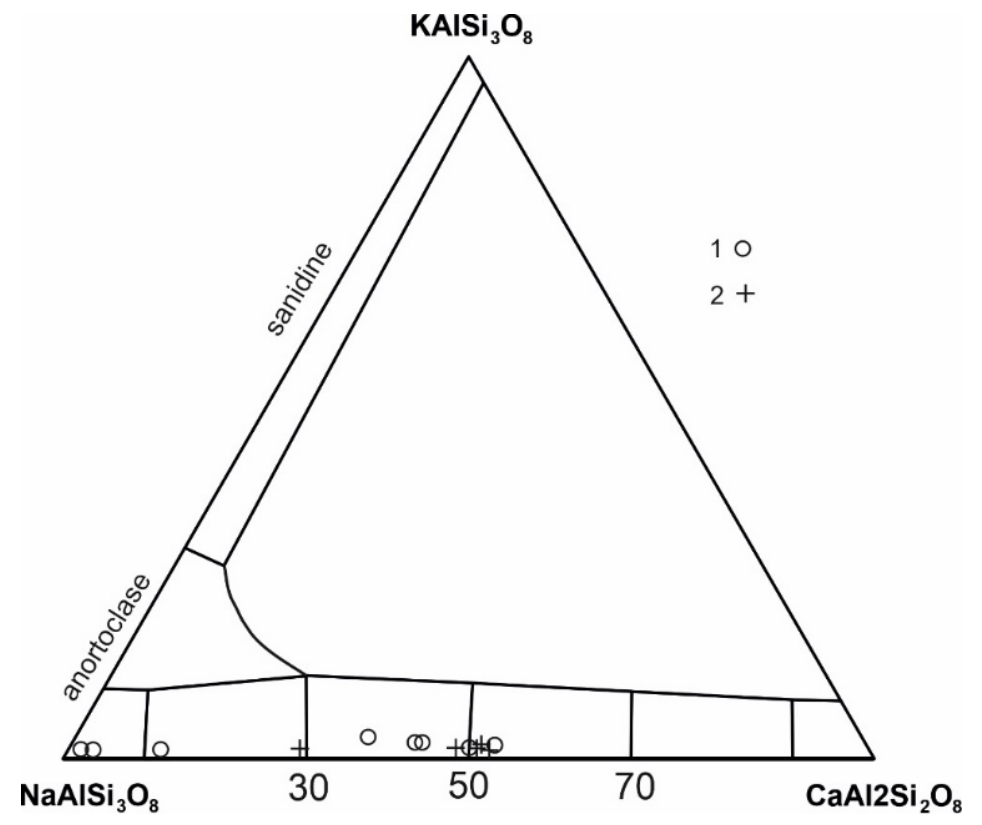

Figure 6. Classification diagram of studied plagioclases. 1—sample M-1; 2-sample M-9.

Pumpellyite was detected in the central parts of altered plagioclase and along the fissures of crystals, respectively (Figure 2a,d, Table 4). Pumpellyite and prehnite together with albite form a younger loaded mineral association. At the same time, the presence of pumpellyite and prehnite points to postmagmatic metamorphic processes. Their age and spatial relationships were studied by Vrána and Vozár [33] and Spišiak and Pitoňák [53].

Rare small grains of $\mathrm{Cr}$-spinel have been observed in the gabbro-diorite dyke (sample M-8). Spinel grains probably formed part of the primary mineral association (clinopyroxenes, plagioclases, pargasite). Due to subsequent postmagmatic processes, rocks were altered, and spinels are found in the environment of secondary minerals: actinolite, and/or albite, prehnite, pumpellyite (rarely calcite) (Figure 7). The composition of the studied spinels is similar, and they are characterized by high contents of $\mathrm{Al}_{2} \mathrm{O}_{3}, \mathrm{Cr}_{2} \mathrm{O}_{3}$, and FeOtot (Table 6). The minor differences in spinel composition are presumably caused by the mineral environment in which they occur. Overall, we can divide the studied spinels according to the type of surrounding minerals into: (1) spinels in actinolites and (2) spinels in associations-albite, prehnite, and chlorite. Compared to the second group, the spinels in the first group (in actinolites) have a higher $\mathrm{Fe}$ (and $\mathrm{Zn}$ ) content and lower $\mathrm{Al}, \mathrm{Cr}$, and $\mathrm{Mg}$ (less Ti). The most significant differences are in the contents of $\mathrm{Mg}$ and $\mathrm{Fe}^{2+}$ (Figure 8A). In the figure, we can also observe a significant negative correlation between these elements. The composition of spinels corresponds to spinels from volcanic rocks [50,51], so they are not relics of spinels from upper mantle (Figure 8B). 

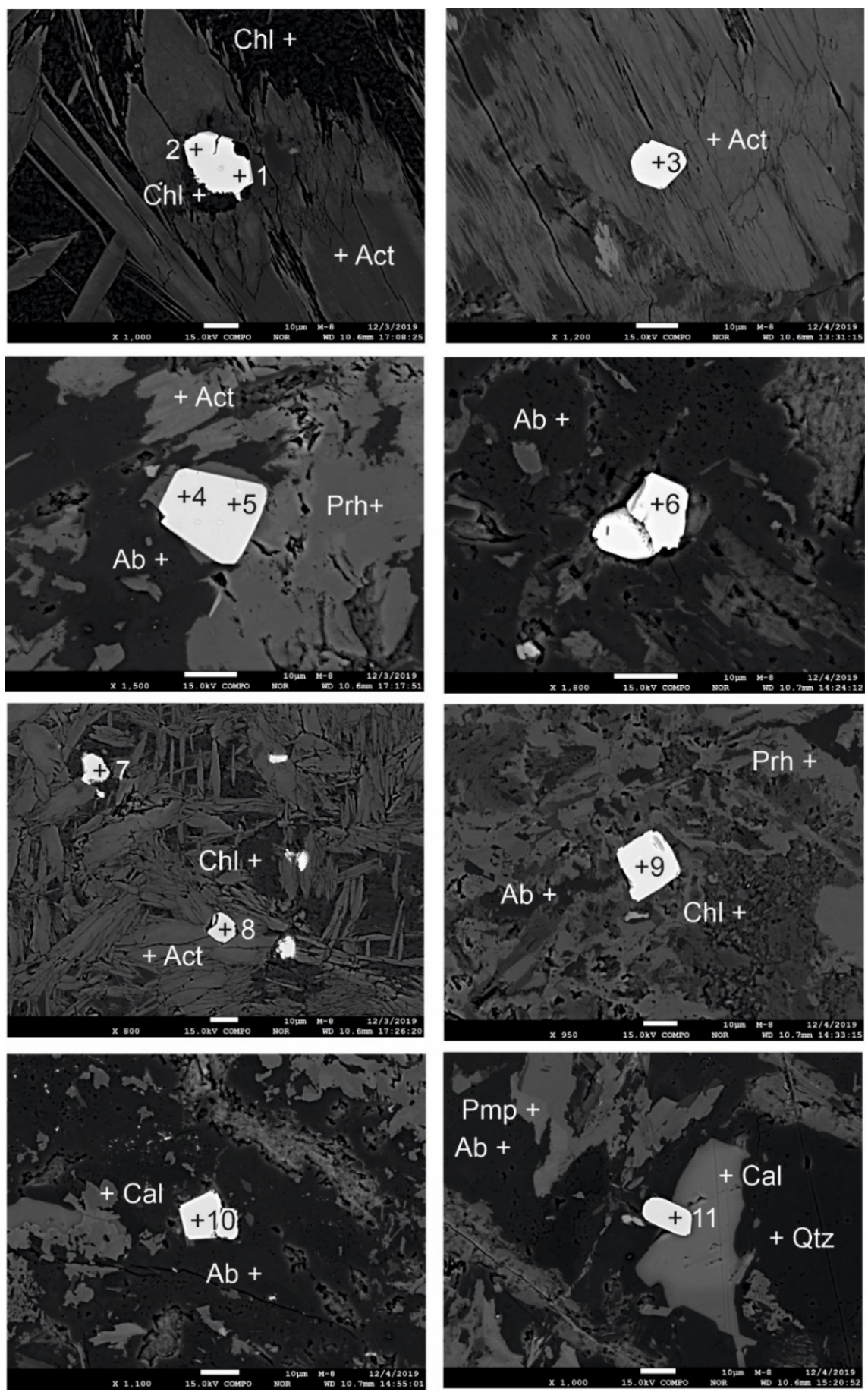

Figure 7. Back scattered electron (BSE) images of basalts from Malužiná; Act = actinolite, $\mathrm{Chl}=$ chlorite, $\mathrm{Ab}=$ albite, $\mathrm{Prh}=$ prehnite, $\mathrm{Pmp}=$ pumpellyite, Cal = calcite, $\mathrm{Spl}=$ spinels; the numbers in figures correspond to those in Tables $2-5$. 
Table 6. Selected analyses of spinels.

\begin{tabular}{cccccccccccc}
\hline Anal. $\mathbf{~}$. & $\mathbf{1}$ & $\mathbf{2}$ & $\mathbf{3}$ & $\mathbf{4}$ & $\mathbf{5}$ & $\mathbf{6}$ & $\mathbf{7}$ & $\mathbf{8}$ & $\mathbf{9}$ & $\mathbf{1 0}$ & $\mathbf{1 1}$ \\
\hline $\mathrm{SiO}_{2}$ & 0.09 & 0.05 & 0.11 & 0.08 & 0.07 & 0.138 & 0.11 & 0.08 & 0.00 & 0.05 & 0.08 \\
\hline $\mathrm{TiO}_{2}$ & 1.11 & 0.86 & 1.53 & 1.93 & 1.93 & 1.322 & 0.83 & 0.66 & 2.31 & 1.86 & 1.85 \\
\hline $\mathrm{Al}_{2} \mathrm{O}_{3}$ & 12.76 & 13.19 & 9.29 & 20.71 & 20.39 & 16.265 & 16.55 & 15.33 & 17.47 & 20.28 & 20.42 \\
\hline $\mathrm{Cr}_{2} \mathrm{O}_{3}$ & 27.59 & 27.81 & 30.24 & 32.86 & 33.70 & 36.020 & 29.00 & 29.35 & 34.45 & 33.68 & 32.66 \\
\hline $\mathrm{FeO}$ & 26.98 & 26.47 & 27.43 & 17.91 & 18.25 & 13.062 & 25.98 & 25.70 & 18.46 & 17.44 & 17.08 \\
\hline $\mathrm{Fe}_{2} \mathrm{O}_{3}$ & 25.97 & 25.26 & 26.17 & 14.14 & 13.42 & 21.435 & 20.60 & 21.84 & 15.26 & 14.06 & 14.11 \\
\hline $\mathrm{MnO}$ & 0.41 & 0.46 & 0.45 & 0.34 & 0.34 & 0.380 & 0.39 & 0.40 & 0.30 & 0.32 & 0.37 \\
\hline $\mathrm{MgO}$ & 3.69 & 3.87 & 2.95 & 10.67 & 10.44 & 8.556 & 4.63 & 4.71 & 9.89 & 10.93 & 10.91 \\
\hline $\mathrm{ZnO}$ & 0.31 & 0.33 & 0.19 & 0.14 & 0.01 & 0.10 & 0.46 & 0.31 & 0.12 & 0.14 & 0.16 \\
\hline $\mathrm{V}_{2} \mathrm{O} 5$ & 0.20 & 0.24 & 0.34 & 0.29 & 0.26 & 0.23 & 0.23 & 0.19 & 0.31 & 0.22 & 0.23 \\
\hline $\mathrm{NiO}$ & 0.02 & 0.11 & 0.05 & 0.00 & 0.02 & 0.00 & 0.06 & 0.08 & 0.01 & 0.04 & 0.01 \\
\hline total & 99.13 & 98.62 & 98.75 & 99.06 & 98.83 & 97.51 & 98.83 & 98.65 & 98.58 & 99.01 & 97.89 \\
\hline & & & & Formula based $0 n$ & 4 oxygens & & & & \\
\hline $\mathrm{Si}$ & 0.003 & 0.002 & 0.004 & 0.003 & 0.002 & 0.005 & 0.004 & 0.003 & 0.000 & 0.002 & 0.002 \\
\hline $\mathrm{Ti}$ & 0.029 & 0.023 & 0.041 & 0.047 & 0.047 & 0.033 & 0.022 & 0.017 & 0.058 & 0.045 & 0.045 \\
\hline $\mathrm{Al}$ & 0.526 & 0.545 & 0.393 & 0.795 & 0.779 & 0.642 & 0.674 & 0.622 & 0.681 & 0.772 & 0.785 \\
\hline $\mathrm{Cr}$ & 0.763 & 0.771 & 0.859 & 0.847 & 0.864 & 0.953 & 0.793 & 0.799 & 0.901 & 0.860 & 0.842 \\
\hline $\mathrm{Fe}{ }^{3+}$ & 0.681 & 0.663 & 0.704 & 0.339 & 0.324 & 0.329 & 0.527 & 0.563 & 0.375 & 0.338 & 0.342 \\
\hline $\mathrm{Fe}{ }^{2+}$ & 0.786 & 0.772 & 0.820 & 0.478 & 0.489 & 0.600 & 0.739 & 0.736 & 0.504 & 0.466 & 0.460 \\
\hline $\mathrm{Mn}$ & 0.012 & 0.014 & 0.014 & 0.009 & 0.009 & 0.011 & 0.011 & 0.012 & 0.008 & 0.009 & 0.010 \\
\hline $\mathrm{Mg}$ & 0.193 & 0.202 & 0.158 & 0.518 & 0.504 & 0.427 & 0.239 & 0.242 & 0.488 & 0.526 & 0.530 \\
\hline & & & & & & & & & & &
\end{tabular}
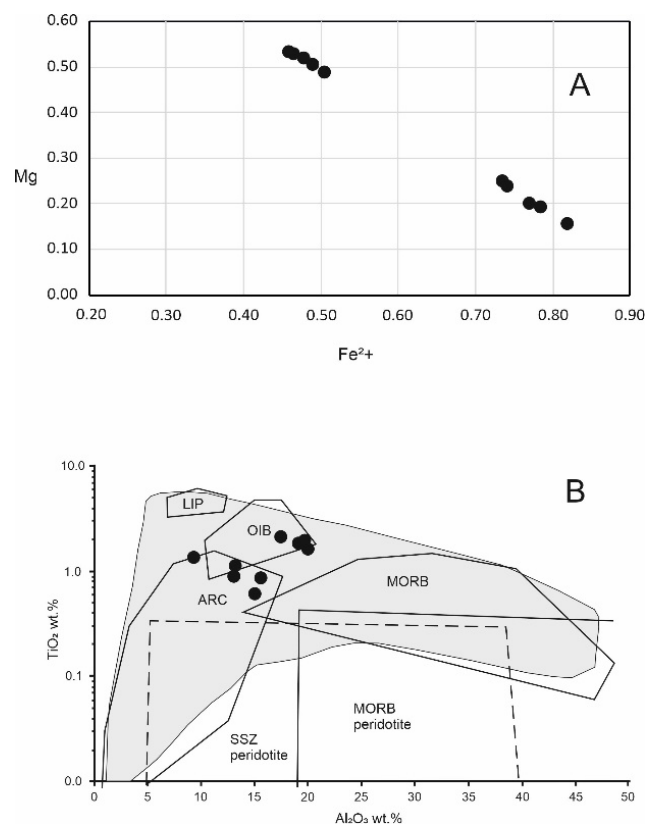

Figure 8. (A) $\mathrm{Mg}$ vs. $\mathrm{Fe}^{2+}$ plot; (B) $\mathrm{TiO}_{2}$ versus $\mathrm{Al}_{2} \mathrm{O}_{3}$ plot for studied spinels. Large Igneous Province (LIP), Ocean Island Basalts (OIB), Island Arc magmas (ARC), Mid Ocean Ridge Basalts (MORB), volcanic rocks (grey field), Suprasubduction Zone (SSZ), MORB and peridotite fields after Lenaz et al. [54] and Kamenetsky et al. [55]. 


\section{Geochemistry}

Seven samples were studied for chemical composition (Table 7). $\mathrm{SiO}_{2}$ content in the samples varied from 44 to $54 \mathrm{wt} . \%$. The mg\# number was low to moderate, ranging from 25 to 68 . The contents of $\mathrm{TiO}_{2}$ correspond accordingly (1.2-2.9 wt.\%). Based on TAS classification, the rock studied corresponds with the basalt and basaltic andesite field of subalkaline rocks (Figure 9A). However, strong secondary alteration increased alkali contents and shifted sample composition within the field of alkaline rocks. As alkali are highly mobile during super-imposed hydrothermal processes and regional metamorphism, the high field strength elements (HFSE) $\mathrm{Zr}, \mathrm{Nb}, \mathrm{Y}$, and $\mathrm{TiO}_{2}$ were used for rock classification. Based on Winchester and Floyd [56] $\mathrm{Nb} / \mathrm{Y}$ vs. $\mathrm{Zr} / \mathrm{TiO}_{2}$ discrimination diagram, the analysed rocks form a coherent group within the andesite/basalt field (Figure 9B). For a more precise geotectonic classification of the studied rocks, different ternary diagrams were used (Figure 10A [57]; Figure 10B [58]). In both diagrams, the analyses of the studied rocks fall within the field of calc-alkali, and/or continental basalts. The REE normalized curve (Figure 11A [59]) shows a slight enrichment in light REE, with no significant HREE fractionation. The REE pattern is slightly different in M-3 sample, with the lowest LREE contents; this may be due to a different mineral composition, which is reflected in a significantly lower $\mathrm{CaO}$ content compared to other samples. Nevertheless, negative Eu anomalies are slight to negligible for all the samples (0.72-0.97). In the primitive mantle normalized plots (Figure 11B), the analysed samples show a marked depletion of $\mathrm{Rb}$, $\mathrm{Ba}, \mathrm{Nb}, \mathrm{Ta}$, and $\mathrm{Sr}$, as well as a slight depletion of Ti and HREE. On the other hand, the enrichment in Th, $\mathrm{U}$, accompanied also by a slight enrichment of $\mathrm{P}, \mathrm{Nd}$, and $\mathrm{Zr}$, is observed.

Table 7. Chemical composition of studied samples.

\begin{tabular}{cccccccc}
\hline Sample & $\mathbf{M - 1}$ & $\mathbf{M - 1 A}$ & $\mathbf{M - 2}$ & $\mathbf{M - 3}$ & $\mathbf{M - 4}$ & $\mathbf{M - 5}$ & M-7 \\
\hline $\mathrm{SiO}_{2}$ & 50.24 & 49.14 & 50.54 & 49.57 & 44.52 & 53.92 & 53.42 \\
\hline $\mathrm{TiO}_{2}$ & 1.42 & 2.09 & 2.14 & 1.61 & 1.26 & 1.20 & 2.46 \\
\hline $\mathrm{Al}_{2} \mathrm{O}_{3}$ & 15.87 & 16.80 & 15.98 & 17.88 & 17.26 & 15.24 & 16.72 \\
\hline $\mathrm{Fe}_{2} \mathrm{O}_{3}{ }^{*}$ & 7.12 & 10.88 & 10.21 & 9.95 & 6.37 & 9.65 & 11.36 \\
\hline $\mathrm{Cr}_{2} \mathrm{O}_{3}$ & 0.02 & 0.01 & $<0.002$ & 0.01 & 0.02 & 0.01 & $<0.002$ \\
\hline $\mathrm{CaO}$ & 8.33 & 7.58 & 5.88 & 1.12 & 8.31 & 4.55 & 0.69 \\
\hline $\mathrm{MnO}$ & 0.12 & 0.17 & 0.13 & 0.09 & 0.10 & 0.09 & 0.04 \\
\hline $\mathrm{MgO}$ & 5.46 & 5.61 & 1.68 & 9.07 & 6.07 & 3.40 & 5.77 \\
\hline $\mathrm{Na}{ }_{2} \mathrm{O}$ & 4.21 & 3.53 & 7.31 & 4.45 & 2.83 & 6.66 & 4.79 \\
\hline $\mathrm{K}_{2} \mathrm{O}$ & 0.88 & 1.35 & 0.27 & 0.46 & 1.63 & 0.14 & 0.17 \\
\hline $\mathrm{P}_{2} \mathrm{O}_{5}$ & 0.24 & 0.32 & 0.49 & 0.18 & 0.20 & 0.21 & 0.43 \\
\hline $\mathrm{LOl}$ & 5.90 & 2.20 & 5.20 & 5.40 & 11.20 & 4.80 & 3.90 \\
\hline $\mathrm{Total}$ & 99.81 & 99.68 & 99.83 & 99.79 & 99.77 & 99.87 & 99.75 \\
\hline $\mathrm{TOT} / \mathrm{C}$ & 0.74 & 0.03 & 1.11 & 0.09 & 1.77 & 0.69 & $<0.02$ \\
\hline $\mathrm{TOT} / \mathrm{S}$ & $<0.02$ & 0.02 & $<0.02$ & $<0.02$ & $<0.02$ & $<0.02$ & $<0.02$ \\
\hline $\mathrm{Sc}$ & 30.00 & 25.00 & 20.00 & 26.00 & 23.00 & 18.00 & 25.00 \\
\hline $\mathrm{Ba}$ & 152.00 & 697.00 & 87.00 & 51.00 & 206.00 & 48.00 & 439.00 \\
\hline $\mathrm{Be}$ & 2.00 & 2.00 & 1.00 & $<1$ & $<1$ & $<1$ & 2.00 \\
\hline $\mathrm{Co}$ & 28.20 & 36.8 & 21.10 & 35.00 & 34.40 & 24.50 & 26.80 \\
\hline $\mathrm{Cs}$ & 2.30 & 2.90 & 0.40 & 2.50 & 5.10 & 0.40 & 0.70 \\
\hline $\mathrm{Ga}$ & 16.90 & 19.20 & 19.20 & 17.40 & 15.60 & 14.50 & 20.00 \\
\hline $\mathrm{Hf}$ & 5.20 & 5.40 & 6.80 & 3.90 & 3.90 & 4.30 & 6.30 \\
\hline & & & & & & \\
\hline
\end{tabular}


Table 7. Cont.

\begin{tabular}{|c|c|c|c|c|c|c|c|}
\hline Sample & M-1 & M-1A & M-2 & M-3 & M-4 & M-5 & M-7 \\
\hline $\mathrm{Nb}$ & 9.78 & 9.70 & 8.60 & 3.50 & 7.40 & 6.30 & 11.10 \\
\hline $\mathrm{Rb}$ & 35.10 & 43.70 & 8.20 & 11.30 & 59.10 & 4.40 & 5.40 \\
\hline Sn & 1.00 & 2.00 & 2.00 & 1.00 & 1.00 & 2.00 & 2.00 \\
\hline $\mathrm{Sr}$ & 253.10 & 375.50 & 108.00 & 133.60 & 59.80 & 224.80 & 57.90 \\
\hline Ta & 0.60 & 0.60 & 0.70 & 0.30 & 0.50 & 0.40 & 0.80 \\
\hline Th & 5.30 & 3.00 & 3.30 & 1.60 & 3.90 & 3.30 & 6.40 \\
\hline $\mathrm{U}$ & 1.20 & 1.00 & 1.20 & 0.40 & 1.10 & 0.70 & 2.80 \\
\hline $\mathrm{V}$ & 187.00 & 218.00 & 137.00 & 200.00 & 170.00 & 166.00 & 262.00 \\
\hline W & 6.60 & 6.00 & 7.60 & 4.20 & 2.30 & 5.60 & 11.90 \\
\hline $\mathrm{Zr}$ & 200.20 & 224.10 & 271.80 & 149.00 & 153.40 & 147.50 & 231.60 \\
\hline $\mathrm{Y}$ & 33.60 & 39.60 & 53.20 & 24.40 & 26.00 & 23.60 & 45.30 \\
\hline $\mathrm{La}$ & 23.80 & 17.30 & 17.70 & 8.10 & 21.00 & 15.70 & 19.90 \\
\hline $\mathrm{Ce}$ & 53.50 & 41.60 & 44.70 & 21.30 & 47.30 & 38.90 & 50.20 \\
\hline $\operatorname{Pr}$ & 6.69 & 5.73 & 6.36 & 2.95 & 5.82 & 5.22 & 7.04 \\
\hline $\mathrm{Nd}$ & 27.90 & 25.70 & 29.70 & 13.10 & 24.60 & 21.20 & 30.90 \\
\hline $\mathrm{Sm}$ & 6.20 & 6.46 & 7.80 & 3.54 & 4.99 & 4.51 & 7.30 \\
\hline $\mathrm{Eu}$ & 1.72 & 1.88 & 2.37 & 0.91 & 1.63 & 1.43 & 2.05 \\
\hline $\mathrm{Gd}$ & 6.45 & 7.19 & 9.56 & 4.19 & 5.01 & 4.74 & 8.40 \\
\hline $\mathrm{Tb}$ & 0.99 & 1.11 & 1.49 & 0.69 & 0.76 & 0.71 & 1.33 \\
\hline Dy & 6.11 & 7.07 & 9.35 & 4.48 & 4.57 & 4.46 & 8.16 \\
\hline Ho & 1.17 & 1.43 & 1.88 & 0.93 & 0.91 & 0.99 & 1.67 \\
\hline Er & 3.36 & 4.05 & 5.50 & 2.73 & 2.61 & 2.61 & 4.76 \\
\hline $\mathrm{Tm}$ & 0.51 & 0.60 & 0.79 & 0.42 & 0.37 & 0.40 & 0.70 \\
\hline $\mathrm{Yb}$ & 3.19 & 3.77 & 5.05 & 2.59 & 2.36 & 2.48 & 4.31 \\
\hline $\mathrm{Lu}$ & 0.50 & 0.58 & 0.78 & 0.42 & 0.35 & 0.40 & 0.68 \\
\hline $\mathrm{Ni}$ & 41.00 & 50.00 & $<20$ & 32.00 & 81.00 & 35.00 & $<20$ \\
\hline
\end{tabular}



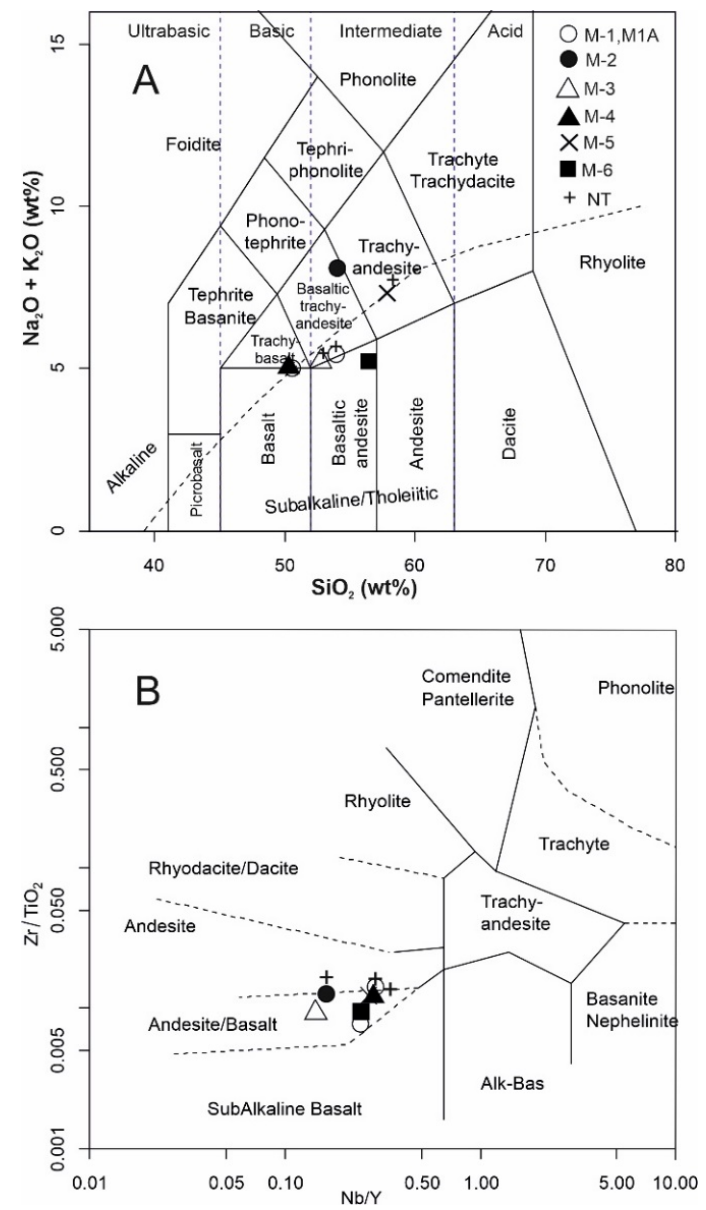

Figure 9. (A) TAS plot [60]; (B) $\mathrm{Nb} / \mathrm{Y}$ vs. $\mathrm{Zr} / \mathrm{TiO}_{2}$ plot for studied basalts [56]. Sample M-1-M7 from Table 7, NT—analyses of paleobasalts from Vozár et al. [61].

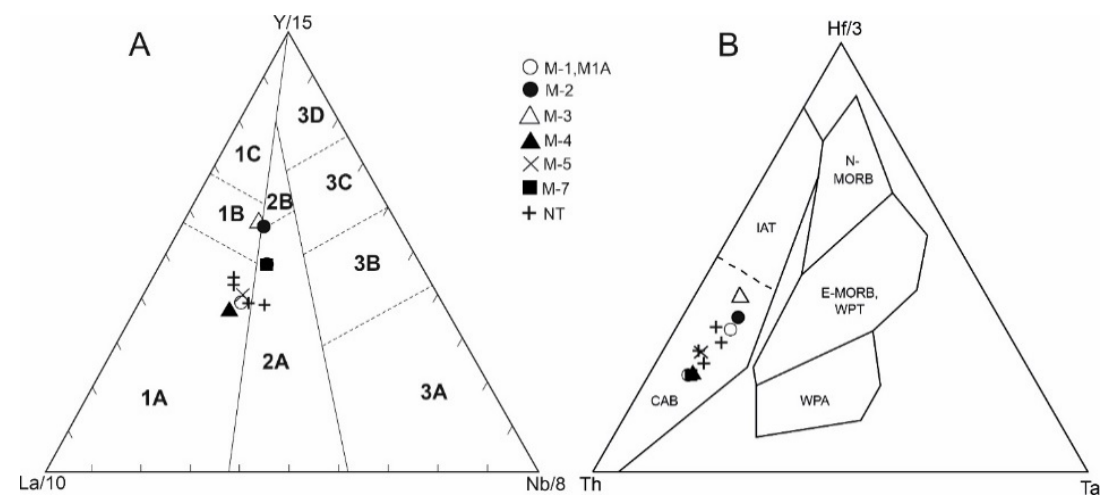

Figure 10. (A) The La-Y-Nb: discrimination diagram for basalts [58] Field 1-volcanic arc basalts, field 2-continental basalts and field 3-oceanic basalts. The subdivision of the fields as follows: $1 \mathrm{~A}=$ calc-alkaline basalts, $1 \mathrm{C}=$ volcanic arc tholeiites, $1 \mathrm{~B}=$ overlap is between $1 \mathrm{~A}$ and $1 \mathrm{C}, 2 \mathrm{~A}=$ continental basalts, $2 \mathrm{~B}=$ back-are basin basalts, $3 \mathrm{~A}=$ alkaline basalts from intercontinental rift, 3B, 3C = E-type MORB (enriched MORB), 3D = N type- MORB; B) The Th-Hf-Ta: discrimination diagram for basalts [57]. (B) The field are defined as follows: N-MORB (normal Mid Ocean Ridge Basalt), E-MORB and WPT (within-plate tholeiites), WPA (within-plate alkali basalts), IAT (Island arc tholeiites, Hf/Th > 3.0) and CAB (calc-alkali basalts; Hf/Th < 3.0). The broken lines indicate transitional zones between basalt types. Sample M-1-M7 from Table 7, NT-analyses of paleobasalts from Vozár et al. [61]. 

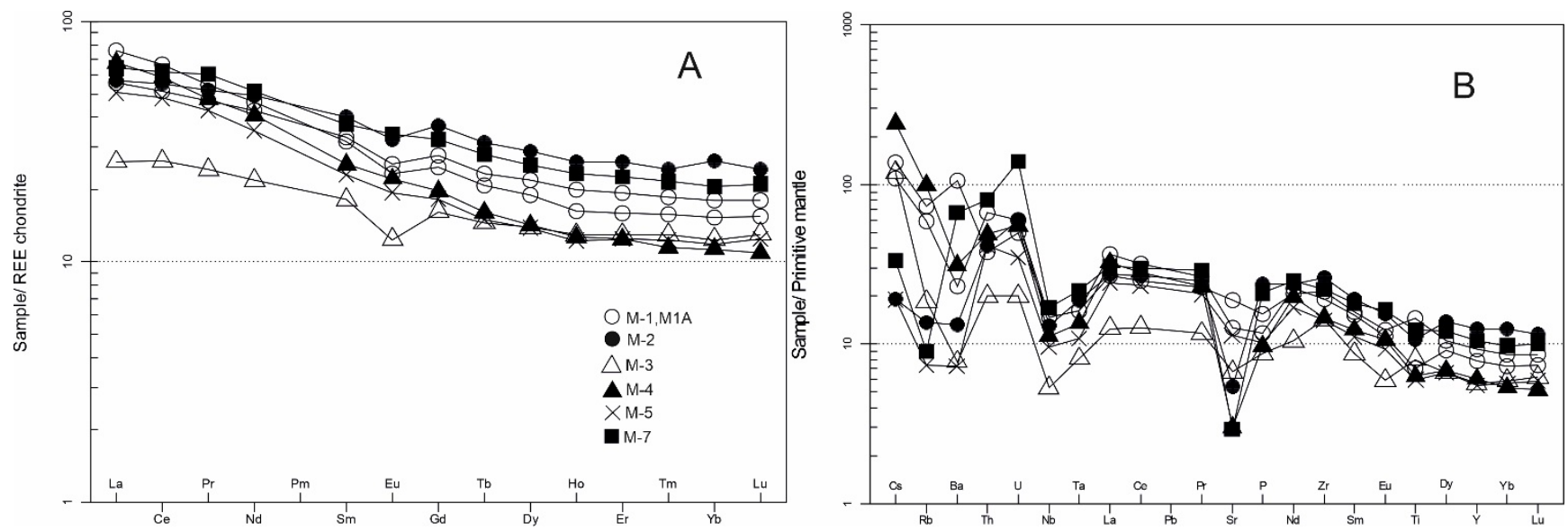

Figure 11. (A) REE concentration normalized to the chondrite composition [59]; (B) Trace element concentrations normalized to the composition of the primitive mantle [62]. Sample M-1-M7 from Table 7.

The $\mathrm{Sr}, \mathrm{Nd}$, and $\mathrm{Pb}$ isotopic compositions were determined in five samples (Table 8, whole rocks) and together with the three previously published results [61] the whole set is represented by eight samples. Two samples from the diorite dykes and three samples from the first and second eruption phases were analysed. The Nd-Sr isotope signature of the studied basalts are scattered around the value of ${ }^{143} \mathrm{Nd} /{ }^{144} \mathrm{Nd}$ for CHUR, where ${ }^{143} \mathrm{Nd}^{144} \mathrm{Nd}$ ratios are relatively stable and ${ }^{87} \mathrm{Sr} /{ }^{86} \mathrm{Sr}$ values are relatively varied. (Figure 12). Some of the points lie in field IV, and/or its vicinity, indicating a relatively high proportion of crustal contamination in the formation of these basalts. The study of $\mathrm{Pb}$ isotopes gives us similar conclusions. The diagram (Figure 13) illustrates the isotopic ratio of ${ }^{207} \mathrm{~Pb} /{ }^{204} \mathrm{~Pb}$ vs. ${ }^{206} \mathrm{~Pb} /{ }^{204} \mathrm{~Pb}$. The basalt analyses lie in the field of EMII, and/or they partially overlap with the Saxo-Thuringian upper crust fields. Compared to the MORB field, they have higher ${ }^{207} \mathrm{~Pb}^{204} \mathrm{~Pb}$. The EMII field is defined as a mantle source enriched with crustal materials $[63,64]$. Crustal materials can be incorporated into mantle material in different ways $[65,66]$. This position indicates a relatively high degree of crustal contamination. This is especially evident from ${ }^{206} \mathrm{~Pb} /{ }^{204} \mathrm{~Pb}$ isotope ratios, which correspond to values around 19. This $\mathrm{Pb}$ isotope ratio is considered to be the most sensitive indicator of crustal contamination.

Table 8. $\mathrm{Sr}, \mathrm{Nd}$, and $\mathrm{Pb}$ isotope analytical data from the studied samples.

\begin{tabular}{cccccc}
\hline Sample & M-1 & M-3 & M-4 & M-5 & M-7 \\
\hline${ }^{206} \mathrm{~Pb} /{ }^{204} \mathrm{~Pb}$ & 18.92209939 & 18.7368185 & 19.03282702 & 18.57438457 & 19.14024785 \\
\hline $\pm 2 \mathrm{~s}+$ & 0.011112606 & 0.01491399 & 0.012461895 & 0.010005923 & 0.019462799 \\
\hline${ }^{207} \mathrm{~Pb} /{ }^{204} \mathrm{~Pb}$ & 15.66053538 & 15.6515818 & 15.66947086 & 15.6394058 & 15.6842099 \\
\hline $\pm 2 \mathrm{~s}+$ & 0.010983742 & 0.014073549 & 0.011949346 & 0.010340995 & 0.017133993 \\
\hline${ }^{208} \mathrm{~Pb} /{ }^{204} \mathrm{~Pb}$ & 39.09866217 & 38.74599643 & 39.10893752 & 38.5879748 & 39.07321516 \\
\hline $\pm 2 \mathrm{~s}+$ & 0.032498962 & 0.038868704 & 0.034110976 & 0.030599558 & 0.045900435 \\
\hline${ }^{207} \mathrm{~Pb} /{ }^{206} \mathrm{~Pb}$ & 0.827633773 & 0.83534005 & 0.823288311 & 0.841989613 & 0.819437825 \\
\hline $\pm 2 \mathrm{~s}+$ & 0.000174809 & 0.000224997 & 0.000184886 & 0.000177841 & 0.000195686 \\
\hline${ }^{208} \mathrm{~Pb} /{ }^{206} \mathrm{~Pb}$ & 2.066304938 & 2.067915649 & 2.054823635 & 2.077492136 & 2.04142499 \\
\hline $\pm 2 \mathrm{~s}+$ & 0.000776447 & 0.000847523 & 0.000732947 & 0.000759999 & 0.000767098 \\
\hline${ }^{87} \mathrm{Sr} /{ }^{86} \mathrm{Sr}$ & 0.707907300 & 0.706442300 & 0.713264800 & 0.706534300 & 0.708874500 \\
\hline$+/-\mathrm{abs}$ & $4.95535 \times 10^{-6}$ & $6.35798 \times 10^{-6}$ & $4.27959 \times 10^{-6}$ & $4.23921 \times 10^{-6}$ & $4.96212 \times 10^{-6}$ \\
\hline${ }^{143} \mathrm{Nd} /{ }^{144} \mathrm{Nd}$ & 0.512546651 & 0.512868216 & 0.51259686 & 0.512701087 & 0.512593255 \\
\hline
\end{tabular}


Table 8. Cont.

\begin{tabular}{cccccc}
\hline Sample & M-1 & M-3 & M-4 & M-5 & M-7 \\
\hline$+/-$ abs & $5.63801 \times 10^{-6}$ & $6.15442 \times 10^{-6}$ & $5.63857 \times 10^{-6}$ & $5.63971 \times 10^{-6}$ & $3.58815 \times 10^{-6}$ \\
\hline Age $(\mathrm{Ma})$ & 265 & 265 & 265 & 265 & 265 \\
\hline$\varepsilon \mathrm{Nd}$ & 0.33 & 5.62 & 1.7 & 3.54 & 0.95 \\
\hline
\end{tabular}

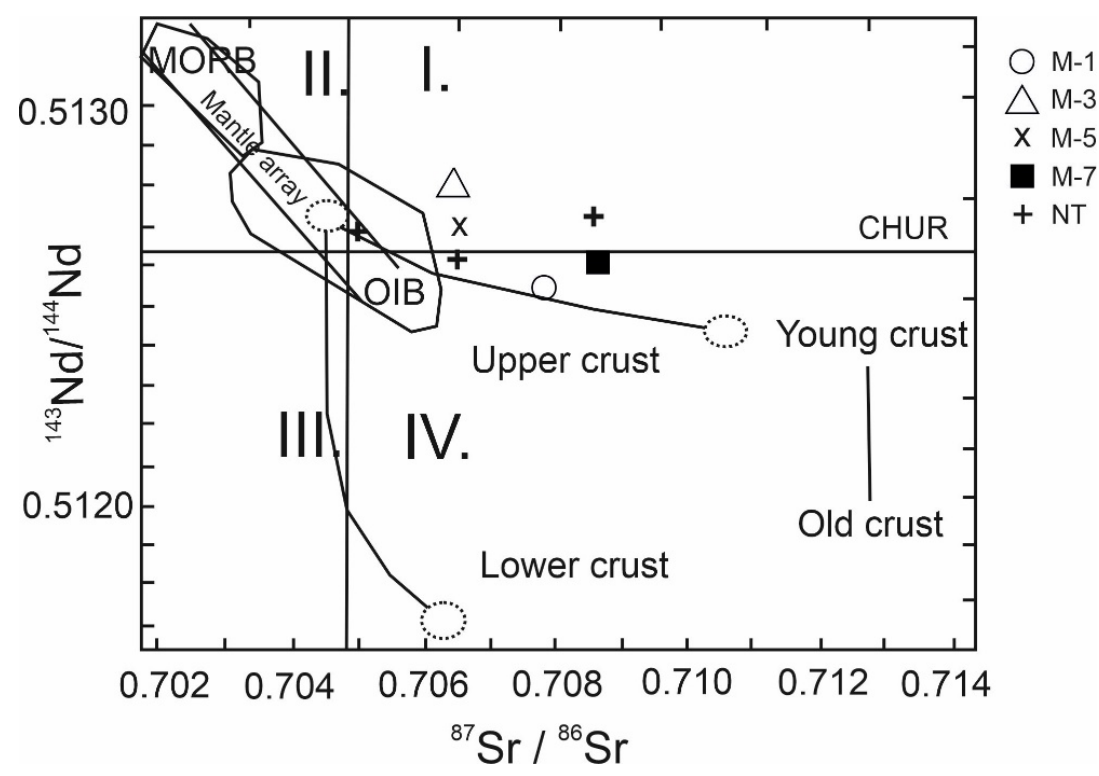

Figure 12. ${ }^{143} \mathrm{Nd} /{ }^{144} \mathrm{Nd}$ vs. ${ }^{87} \mathrm{Sr} /{ }^{86} \mathrm{Sr}$ discriminant diagram, CHUR-chondrite uniform reservoir; OIB — oceanic island basalt; MORB — mid ocean ride basalts, according to Faure [67]. Sample M-1— M7 from Table 8, NT—analyses of paleobasalts from Vozár et al. [61].

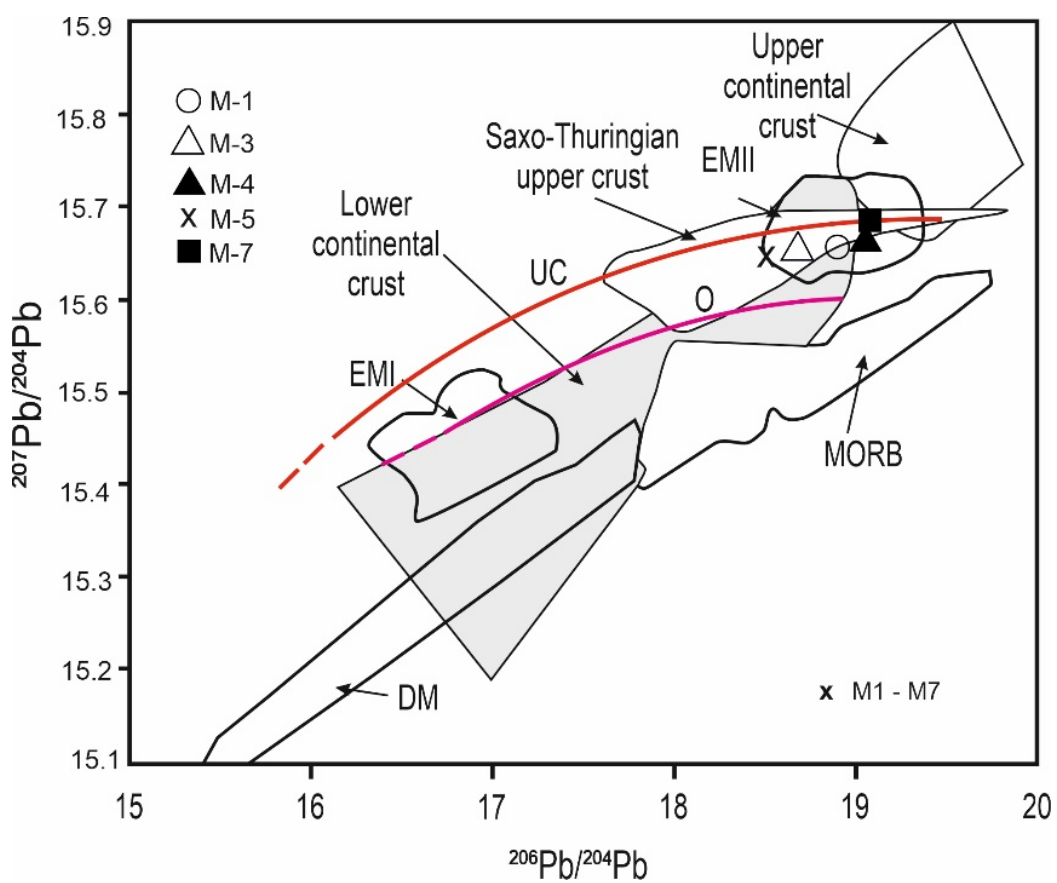

Figure 13. 207Pb/204Pb vs. 206Pb/204Pb in studied basalt; Saxo-Thuringian upper crust are taken from Romer and Hahne [68] and DM, EMI, EMII (from Zindler and Hart [63].Orogene (O) and upper crust (UC) Pb evolution curves are after Zartman and Doe [69].Other fields from Sommer et al. [70]; Sample M-1-M7 from Table 8. 


\section{Discussion and Interpretation}

From the geochemical point of view, the studied Hronicum unit basic rocks can be classified as alkaline-calcium basalts with an affinity for continental basalts. (Figure 10A,B). We used various immobile elements and their ratios to determine the genesis of these rocks (Figure 14). The diagrams show that the rocks studied are not a product of fractional crystallization or a combination of assimilation and fractional crystallization. Their genesis is relatively complex, and it is probably a matter of mixing (contamination) of mantle sources with crust material. If we estimate the proportion of upper crust material and the primary source of N-MORB [71], the studied basalts appear to contain approximately $10 \%$ of crust material.
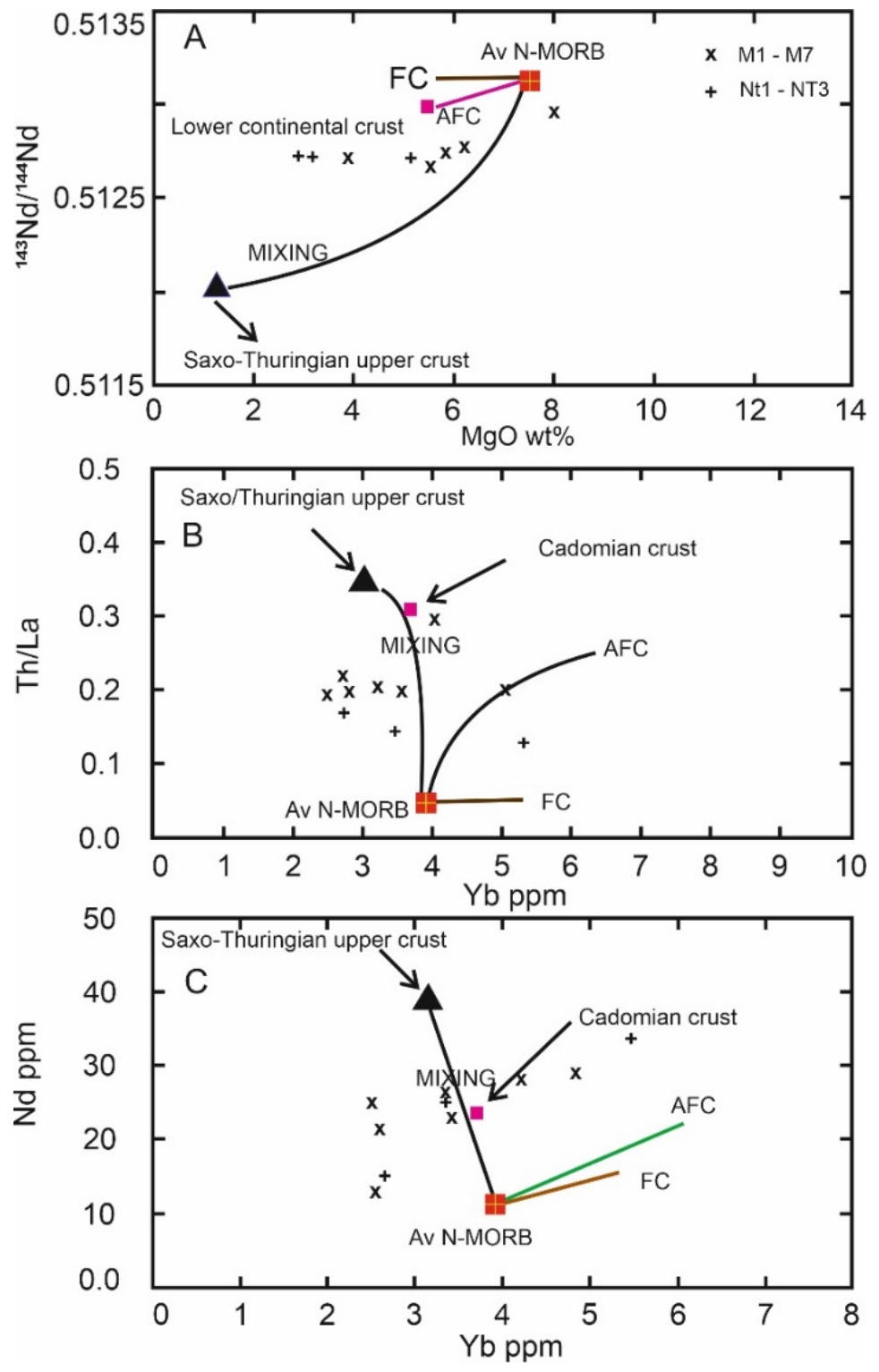

Figure 14. (A) $143 \mathrm{Nd} / 144 \mathrm{Nd}$ vs. $\mathrm{MgO}$; (B) Th/La vs. Yb; (C) Nd vs. Yb plots showing the results of FC, AFC, and mixing modeling results. N-MORB average is from Hofmann [72] and Ito et al. [73]. Saxo-Thuringian upper crust from Romer and Hahne [68], average lower continental crust from Taylor and McLennan [74] and Cadomian crust from Linnemann and Romer [75]. AFC-assimilation with fractional crystallization, FC-fractional crystallization. Others from Abdelfadil et al. [76]. Sample M-1-M7 from Tables 7 and 8, NT—analyses of paleobasalts from Vozár et al. [61]. 
However, the chemical and isotopic compositions of 1st and 2nd eruption phase volcanic rocks from the Malužina Formation do not show greater differences, despite their different stratigraphic position. Perhaps the only difference is the slightly higher value of ${ }^{87} \mathrm{Sr} /{ }^{86} \mathrm{Sr}$ ratio in the volcanics of the 1st eruption phase (samples $\mathrm{M}-7, \mathrm{M}-4$, and NT-2; Table 8), which indicates a relatively high degree of crustal contamination, compared to the 2nd eruption phase. The ${ }^{87} \mathrm{Sr} /{ }^{86} \mathrm{Sr}$ ratio in gabbro-diorite dykes is similar to the volcanics of the 1st eruption phase (samples M-1A and NT-1; Table 8), which could indicate their concurrence. This is also indicated by a slightly higher LILE and LREE enrichment in the volcanics of the 1st eruption phase compared to the 2nd eruption phase (Figure 10A,B). Conversely, ${ }^{143} \mathrm{Nd} /{ }^{144} \mathrm{Nd}$ isotope ratio increases slightly in the volcanics of the 2nd eruption phase compared to the volcanics of the 1st eruption phase, which may also correspond to the differences in the degree of contamination by crustal material. Similarly, variations in ${ }^{206} \mathrm{~Pb} /{ }^{204} \mathrm{~Pb}$ isotope ratio indicate higher values (19.14-19.03) in 1st eruption phase volcanics compared to 2nd eruption phase volcanics (18.37-18.73; Table 8). This trend is probably a result of upper crust contamination, as evidenced by higher ${ }^{206} \mathrm{~Pb} /{ }^{204} \mathrm{~Pb}$ isotope ratios consistent with the increasing ${ }^{87} \mathrm{Sr} /{ }^{86} \mathrm{Sr}$ isotope ratios. A similar trend is shown, for example, by Paraná basalts [77,78].

The values of ${ }^{206} \mathrm{~Pb} /{ }^{204} \mathrm{~Pb}(18.57-19.14),{ }^{207} \mathrm{~Pb} /{ }^{204} \mathrm{~Pb}(15.63-15.68)$ and ${ }^{208} \mathrm{~Pb} /{ }^{204} \mathrm{~Pb}$ (38.58-39.10) are significantly higher in the volcanics of the Malužina Formation compared to depleted mantle (DM) line-MORB basalts, but overall they do not show a large dispersion. We must therefore assume a single magmatic source for both volcanic phases and the dyke complex. As already mentioned, the isotopic composition indicates an affinity with the EM II mantle source. However, the values of $\mathrm{La} / \mathrm{Sm}$ and $\mathrm{Sm} / \mathrm{Yb}(4.2-2.3$ and 1.4-2.1, respectively) are low, indicating derivation from primary mantle and no significant mantle or crust contamination with lithospheric mantle [79]. This would indicate a rapid ascent of magma, which was a reason why it was not contaminated to a greater extent with crust material.

Basic dykes appear in all occurrences of the Hronicum within a dark gray formation referred to as the Nižná Boca Formation. Based on the remains of macroflora [24] and also microflora [25], the Nižná Boca Formation was included in the Stephanian (according to the Regional Stratigraphic Scale of Central Europe in Vozárová and Vozár [3]), and later correlated with the uppermost Pennsylvanian (Lopingian) according to ICS chart (2019) of the stratigraphic scale. Logically, this led to the interpretation that the basic dykes are of pre-Permian age. However, the analysis of detrital zircons [8] showed that the upper age limit of the Nižná Boca Formation should be shifted as far as the Lower Cisuralian (Asselian-Sakmarian). On the other hand, the results of U-Pb LA-ICP-MS dating of apatite point to a younger age of $254 \pm 23 \mathrm{Ma}$ (Figure 15), which (although with a relatively large scatter of values) is evidence of their Permian age. There are more possibilities of interpretation:

- The basic dykes intruded together with the later, second volcanic phase in the Permian, at the time of maximum extension. The age of the second eruption phase in the Malužina Formation is indicated on the basis of the microflora as Thuringien (Planderová [25] according to the regional Central European scale), later correlated with the standard ICS stratigraphic scale as the Wuchiapingian-Changhsingian (period after the Capitanian degree; ca. $265 \mathrm{Ma}$ ), when Pangea breakup began in general and roughly coincides with the Illawara Magnetic Horizon [31,80]. This period is also associated with the so-called "Mid-Permian Episode" by Deroin and Bonin [81], associated with transform strike-slip movement and the onset of maximum extension, leading to Pangea breakage.

- However, it is quite likely that the basic dykes do not have the same age. The extension in the Hronicum sedimentation space took place in several stages and intrusions of dykes could be associated with each stage of extension.

- Another possibility is that during the extrusion period of the second eruption phase, the elements were remobilized and completely overheated, which resulted in recrys- 
tallization of older apatites or rather crystallization of new ones either in basic dykes or in the volcanics of the first eruption phase. This could explain the large variance of ages in dating, but at the same time it would be consistent with the Upper Permian age (Lopingian) of the second eruption phase.

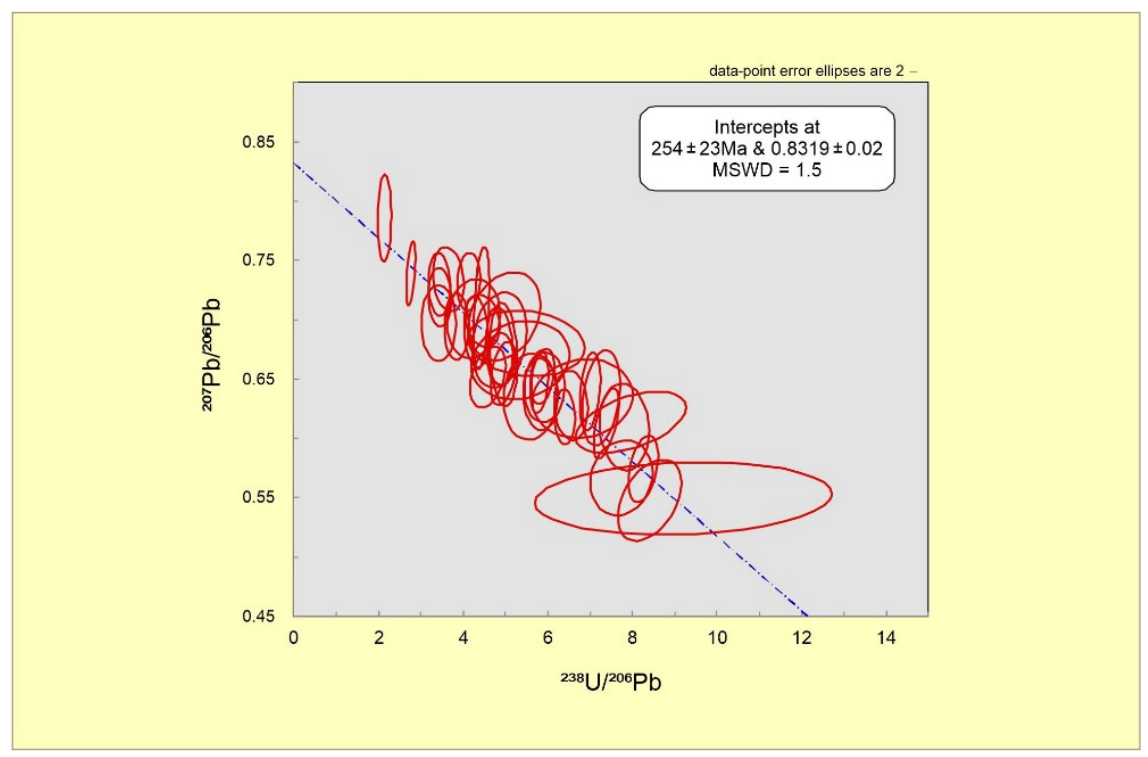

Figure 15. The LA ICP MS analysis of apatites from the Nižná Boca locality; sample M-8.

\section{Conclusions}

1. The basalts of the Malužina Formation are strongly altered; clinopyroxene, plagioclase, and amphibole are locally preserved from the primary minerals. In basalts the predominant textures are intersertal, pilotaxitic, hyalopilitic. Amygdaloid and vesicular structures have been commonly identified.

2. Composition of main rock-forming minerals in different localities is similar. The clinopyroxenes are relatively homogenous in chemical composition, with only rarely observed darker zones in larger grains (Figure 2e) rich in $\mathrm{Mg}$, and/or depleted of Fe. Based on the classification of pyroxenes these Cpx correspond to augite. There are two types of amphibole; the older amphibole coexisted with Cpx and has a composition corresponding to pargasite. The younger amphibole has a composition corresponding to actinolite/tremolite. Biotite is strongly altered and the biotites studied lie in the field of calc-alkaline rocks.

3. Rare small grains of $\mathrm{Cr}$-spinel have been observed in the gabbro-diorite dyke. Spinel grains probably formed part of the primary mineral association (clinopyroxenes, plagioclases, pargasite). The composition of spinels corresponds to spinels from volcanic rocks.

4. From the geochemical point of view, the studied rocks can be classified as alkalinecalcium basalts with affinity for continental basalts. The basalts of the Malužina Formation show a significant depletion of $\mathrm{Nb}$ and Ta compared to $\mathrm{La}$ and $\mathrm{Th}$ when normalized to the primitive mantle. They are also depleted of $\mathrm{P}$, which corresponds to the very low content of apatite in the studied volcanics. These features and the very low content of primary amphibole or apatite in the studied volcanics rather indicate a "dry" magmatic source.

5. Based on the distribution trace elements and $\mathrm{Sr}-\mathrm{Nd}-\mathrm{Pb}$ isotope composition a magmatic source of EM II type can be assumed. The isotopic composition, depletion of $\mathrm{Nb}$ and $\mathrm{Ta}$, and limited variability in the ratios of incompatible elements, indicate only weak crustal assimilation, which could be explained by a rapid ascent of magma to the surface. 
6. The two eruption phases correspond to two significant extension pulses during the development of the Hronicum original sedimentation basin in Permian. The presumed magmatic source of both eruption phases was identical. The differences indicated by the distribution of REE, incompatible elements, as well as $\mathrm{Sr}$ and $\mathrm{Pb}$ isotopes, were due to the relatively higher extents of mantle magma contamination by crust material in the volcanics of the first eruption phase.

Author Contributions: Conceptualization, J.S., A.V. and V.Š.; methodology, J.S., A.V. and J.B.; software, J.B.; validation, J.S. and A.V.; formal analysis, J.V. and Š.F.; investigation and resources, J.S., A.V., J.V., Š.F., V.Š. and J.B.; data curation, J.B.; writing—original draft preparation, J.S. and A.V.; writing-review and editing, J.S. and V.Š.; visualization and supervision, J.S.; project administration, J.S. and V.Š. All authors have read and agreed to the published version of the manuscript.

Funding: Informed consent was obtained from all subjects involved in the study.

Institutional Review Board Statement: Not applicable.

Informed Consent Statement: Not applicable.

Data Availability Statement: Not applicable.

Acknowledgments: This research was supported by grants VEGA 2/0006/19, VEGA 1/0237/18, 033UMB-4/2021 and APVV 19-0065, APVV-0146-16.

Conflicts of Interest: We confirm that the authors have declare no conflict of interest.

\section{References}

1. Ingersoll, R.V.; Busby, C.J. Tectonics of Sedimentary Basins. In Tectonics of Sedimentary Basins; Busby, C.J., Ingersoll, R.V., Eds.; Blackwell Science: Cambridge, UK, 1995; pp. 1-51.

2. Ingersoll, R.V. Tectonic of sedimentary basins, with revised nomenclature. In Tectonics of Sedimentary Basins: Recent Advances; Busby, C., Ingersoll, R.V., Azor, A., Eds.; Blackwell Ltd.: Chichester, UK, 2012; pp. 3-43.

3. Vozárová, A.; Vozár, J. Late Paleozoic in West Carpathians; Monogr, D., Ed.; Štátny Geologický ústav Dionýza Štúra: Bratislava, Slovakia, 1988; pp. 1-314, (In English with Slovak summary).

4. Vozárová, A. Significance of clastic petrofacies for reconstruction of paleotectonic development of the Late Paleozoic in the Western Carpathians. In Sedimentological Problems of the West Carpathians; Sýkora, M., Jablonský, J., Samuel, O., Eds.; Štátny Geologický ústav Dionýza Štúra: Bratislava, Slovakia, 1990; pp. 69-78, (In Slovak with English summary).

5. Vdacny, M.; Vozárová, A.; Vozár, J. Geochemistry of the Permian sandstones from the Malužiná Formation in the Malé Karpaty Mts (Hronic Unit, Western Carpathians, Slovakia): Implications for source-area weathering, provenance and tectonic setting. Geol. Carpathica 2013, 64, 23-38. [CrossRef]

6. Vozárová, A.; Frank, W.; Král', J.; Vozár, J. 40Ar/39Ar dating of detrital mica from the Upper Paleozoic sandstones in the Western Carpathians (Slovakia). Geol. Carpath. 2005, 56, 463-472.

7. Vozárová, A.; Konečný, P.; Vdacny, M.; Vozár, J.; Šarinová, K. Provenance of Permian Malužiná Formation sandstones (Hronicum, Western Carpathians): Evidence from monazite geochronology. Geol. Carpathica 2014, 65, 329-341. [CrossRef]

8. Vozárová, A.; Larionov, A.; Šarinová, K.; Vd’ačný, M.; Lepekhina, E.; Vozár, J.; Lvov, P. Detrital zircons from the Hronicum Carboniferous-Permian sandstones (Western Carpathians, Slovakia): Depositional age and provenance. Int. J. Earth Sci. 2018, 107, 1539-1555. [CrossRef]

9. Vozár, J. Magmatic rocks of the tholeiite series in the Permian of the Choč nappe in the West Carpathians. Miner. Slovaca 1977, 9, 241-258, (In Slovak with English summary).

10. Vozár, J. Rift-related volcanism in the Permian of the Western Carpathians. In Geological Evolution of the Western Carpathians; Grecula, P., Hovorka, D., Putiš, M., Eds.; Mineralia Slovaca: Bratislava, Slovaca, 1997; pp. 225-234.

11. Dostal, J.; Vozár, J.; Keppie, J.D.; Hovorka, D. Permian volcanism in the Central Western Carpathians (Slovakia): Basin-and-Range type rifting in the southern Laurussian margin. Acta Diabetol. 2003, 92, 27-35. [CrossRef]

12. Andrusov, D. Grundriss der Tektonik der Nördlichen Karpaten; Slovak Academy of Sciences: Bratislava, Slovakia, 1968; p. 188.

13. Andrusov, D.; Bystrický, J.; Fusán, O. Outline of the structure of the West Carpathians. In Guide Book for Geological Excursion, Xth Congress of Carpathian-Balkan Association, Czechoslovakia; Dionýz Štúr Geological Institute: Bratislava, Slovakia, 1973; pp. 1-44.

14. Biely, A.; Bezák, V.; Elečko, M.; Gross, P.; Kaličiak, M.; Konečný, V.; Lexa, J.; Mello, J.; Nemčok, J.; Potfaj, M.; et al. Explanation to Geological Map of Slovakia, 1:500,000; Dionýz Štúr Publishers: Bratislava, Slovakia, 1996; pp. 1-76.

15. Biely, A.; Bezák, V.; Elečko, M.; Gross, P.; Kaličiak, M.; Konečný, V.; Lexa, J.; Mello, J.; Nemčok, J.; Potfaj, M.; et al. Geological map of Slovakia; Ministry of the Environment of Slovak Republic; Geological Survey of Slovak Republic: Bratislava, Slovak, 1996.

16. Andrusov, D. Gedanken über das alpin-karpatische Falten-Decken-System. Geol. Sbornik 1960, 6, 171-178, (In Slovak with German summary). 
17. Biely, A.; Fusán, O. Zum Problem der Wurzelzonen der subtatrischen Decken. Geol. Práce Správy 1967, 42, 51-64.

18. Rakús, M.; Potfaj, M.; Vozárová, A. Basic paleogeographic and paleotectonic units of the Western Carpathians. In Geodynamic Development of the Western Carpathians; Rakús, M., Ed.; Štátny geologický ústav Dionýza Štúra: Bratislava, Slovakia, 1998; pp. 15-26.

19. Biely, A.; Bezák, V. (Eds.) Explanation to Geological Map of the Nizke Tatry Mts, 1:50,000; Geological survey of Slovak Republic: Bratislava, Slovak, 1997; pp. 1-232. (In Slovak with English summary).

20. Andrusov, D. Les nappes subtatriques des Carpathes occidentales. Carpatica 1936, 1, 3-50. (In Czech with French summary).

21. Vozárová, A.; Vozár, J. Crystalline complex in the basal part of Choč nappe. Geol. Práce Správy 1979, 72, 195-197. (In Slovak with English summary).

22. Vozárová, A.; Vozár, J. Lithostratigraphical subdivision of Late Paleozoic sequences of the Hronic unit. Miner. Slovaca 1981, 13, 385-403. (In Slovak with English summary).

23. Menning, M.; Hendrich, A. (Eds.) Stratigraphic Table of Germany Compact 2012 (STDKe 2012); Deutsche Stratigraphische Komission: Potsdam, Germany, 2012.

24. Sitár, V.; Vozár, J. Die ersten Makrofloren-Funde in dem Karbon der Choč-Einheit in der Niederen Tatra (Westkarpaten). Geol. Zborn. Geol. Carpath. 1973, 24, 441-448.

25. Planderová, E. Biostratigraphical evaluation of the Carboniferous of the Choč nappe, based on palynology. Geol. Práce Správy 1979, 72, 31-59. (In Slovak with English summary).

26. Vozár, J. Structure of Permian Choč volcanites at the northern slope of the Nízke Tatry Mts. Miner. Petrol. Geochem. Lož. 1974, 1, 1-49. (In Slovak)

27. Planderová, E. Palynological research in the melaphyre series of the Choč unit in the NE part of Nízke Tatry between Spišský Štiavnik and Vikartovce. Geol. Práce Správy 1973, 60, 143-168. (In Slovak with English summary).

28. Planderová, E.; Vozárová, A. Biostratigraphical correlation of Late Paleozoic formations in the West Carpathians. In Correlation of Prevariscan and Variscan Events of the Alpine-Mediterranean Mountain Belt; Sassi, F.P., Varga, I., Eds.; Newsletter 4, IGCP Pr. No. 5, Košice-Padova; State Geological Institute of Dionýz Štúr: Bratislava, Slovakia, 1982; pp. 67-71.

29. Izart, A. The 343 IGCP Working Group. Stratigraphic correlations between the continental and marine Tethyan and Peri-Tethyan basins during the Late Carboniferous and the Early Permian. Geodiversitas 1998, 20, 521-596.

30. Rojkovič, I. Uranium Mineralization in Slovakia; Univerzita Komenského: Bratislava, Slovakia, 1997; pp. 1-117.

31. Menning, M. A Numerical Time Scale for the Permian and Triassic Periods: An Integrated Time Analysis. In The Permian of Northern Pangea; Scholle, P.A., Peryt, T.M., Ulmer-Sholle, D.S., Eds.; Springer: Berlin, Germany, 1995; Volume 1, pp. 77-97.

32. Menning, M. A Permian Time Scale 2000 and correlation of marine and continental sequences using the Illawarra Reversal (265 Ma). In Permian Continental Deposits of Europe and other Areas. Regional Reports and Correlations; Cassinis, G., Ed.; Natura Bresciana: Brescia, Italy, 2001; pp. 355-362.

33. Vrána, S.; Vozár, J. Mineral assemblage pumpellyite-prehnite-quartz facies from Nízke Tatry Mts. Geol. Práce Spráry 1969, 49 , 91-99. (In Slovak)

34. Plašienka, D.; Janák, M.; Hacura, A.; Vrbatovič, P. First illite crystallinity data from Alpine metamorphosed rocks of the Veporicum, Central West Carpathians. Miner. Slovaca 1989, 21, 43-51. (In Slovak with English summary).

35. Šucha, V.; Eberl, D.D. Burial metamorphism of Permian sediments in the Northern Gemeric and Hronic units, West Carpathians. Miner. Slovaca 1992, 24, 399-405.

36. Frei, R.; Polat, A. Chromium isotope fractionation during oxidative weathering-Implications from the study of a Paleoproterozoic (ca. 1.9Ga) paleosol, Schreiber Beach, Ontario, Canada. Precambrian Res. 2013, 224, 434-453. [CrossRef]

37. Wu, T.; Polat, A.; Frei, R.; Fryer, B.J.; Yang, K.-G.; Kusky, T. Geochemistry, Nd, Pb and Sr isotope systematics, and U-Pb zircon ages of the Neoarchean Bad Vermilion Lake greenstone belt and spatially associated granitic rocks, western Superior Province, Canada. Precambrian Res. 2016, 282, 21-51. [CrossRef]

38. Todt, W.; Cliff, R.; Hanser, A.; Hofmann, A.W. Evaluation of a202Pb-205Pb Double Spike for High Precision Lead Isotope Analysis. Large Igneous Prov. 2013, 95, 429-437. [CrossRef]

39. Thirlwall, M. Long-term reproducibility of multicollector Sr and Nd isotope ratio analysis. Chem. Geol. Isot. Geosci. Sect. 1991, 94, 85-104. [CrossRef]

40. Thomson, S.N.; Gehrels, G.E.; Ruiz, J.; Buchwaldt, R. Routine low-damage apatite U-Pb dating using laser ablation-multicollectorICPMS. Geochem. Geophys. Geosystems 2012, 13. [CrossRef]

41. Cochrane, R.; Spikings, R.; Chew, D.; Wotzlaw, J.-F.; Chiaradia, M.; Tyrrell, S.; Schaltegger, U.; Van der Lelij, R. High temperature $\left(>350^{\circ} \mathrm{C}\right)$ thermochronology and mechanisms of Pb loss in apatite. Geochim. Cosmochim. Acta 2014, 127, 39-56. [CrossRef]

42. Chew, D.M.; Donelick, R.A. Combined apatite fission track and U-Pb dating by LA-ICP MS and future trends in apatite provenance analysis. In Quantitative Mineralogy and Microanalysis of Sediments and Sedimentary Rocks; Sylvester, P., Ed.; International Mineralogical Association: Vancouver, Canada, 2012; Chapter 12; pp. 219-248.

43. Schoene, B.; Bowring, S.A. U-Pb systematics of the McClure Mountain syenite: Thermochronological constraints on the age of the 40Ar/39Ar standard MMhb. Contrib. Miner. Pet. 2006, 151, 615-630. [CrossRef]

44. McDowell, F.W.; McIntosh, W.C.; Farley, K. A precise 40Ar-39Ar reference age for the Durango apatite (U-Th)/He and fissiontrack dating standard. Chem. Geol. 2005, 214, 249-263. [CrossRef] 
45. Petrus, J.A.; Kamber, B. VizualAge: A Novel Approach to Laser Ablation ICP-MS U-Pb Geochronology Data Reduction. Geostand. Geoanalytical Res. 2012, 36, 247-270. [CrossRef]

46. Paton, C.; Hellstrom, J.; Paul, B.; Woodhead, J.; Hergt, J. Iolite: Freeware for the visualisation and processing of mass spectrometric data. J. Anal. At. Spectrom. 2011, 26, 2508-2518. [CrossRef]

47. Chew, D.; Petrus, J.; Kamber, B. U-Pb LA-ICPMS dating using accessory mineral standards with variable common $\mathrm{Pb}$. Chem. Geol. 2014, 363, 185-199. [CrossRef]

48. Locock, A. An Excel spreadsheet to classify chemical analyses of amphiboles following the IMA 2012 recommendations. Comput. Geosci. 2014, 62, 1-11. [CrossRef]

49. Morimoto, N.; Fabries, J.; Ferguson, A.K.; Ginzburg, I.V.; Ross, M.; Seifert, F.A.; Zussman, J.; Aoki, K.; Gottardi, G. Nomenclature of Pyroxenes. Miner. Mag. 1988, 52, 535-550. [CrossRef]

50. Hawthorne, C.F.; Oberti, R.; Harlow, G.E.; Maresch, V.W.; Martin, F.R.; Schumacher, C.J.; Welch, D.M. Nomenclature of the amphibole supergroup. Amer. Mineral. 2012, 97, 2031-2048. [CrossRef]

51. Rieder, M.; Cavazzini, G.; D’Yakonov, Y.S.; Frank-Kamenetskii, V.A.; Gottardi, G.; Guggenheim, S.; Koval, P.V.; Müller, G.; Neiva, A.M.R.; Radoslovich, E.W.; et al. Nomenclature of micas. Canad. Mineral. 1998, 36, 905-912. [CrossRef]

52. Abdel-Rahman, A.-F.M. Nature of Biotites from Alkaline, Calc-alkaline, and Peraluminous Magmas. J. Pet. 1994, 35, 525-541. [CrossRef]

53. Spišiak, J.; Pitoňák, P. Pumpellyites from the granodiorites and gneisses of the Nízke Tatry Mts. Crystalline Complex. Acta Geol. Geogr. Univ. Comen. Geol. 1993, 49, 23-27.

54. Lenaz, D.; Kamenetsky, V.S.; Crawford, A.J.; Princivalle, F. Melt inclusions in detrital spinel from the SE Alps (Italy-Slovenia): A new approach to provenance studies of sedimentary basins. Contrib. Miner. Pet. 2000, 139, 748-758. [CrossRef]

55. Kamenetsky, V.; Crawford, A.J.; Meffre, S. Factors Controlling Chemistry of Magmatic Spinel: An Empirical Study of Associated Olivine, Cr-spinel and Melt Inclusions from Primitive Rocks. J. Pet. 2001, 42, 655-671. [CrossRef]

56. Winchester, J.; Floyd, P. Geochemical discrimination of different magma series and their differentiation products using immobile elements. Chem. Geol. 1977, 20, 325-343. [CrossRef]

57. Wood, D. The application of a ThHfTa diagram to problems of tectonomagmatic classification and to establishing the nature of crustal contamination of basaltic lavas of the British Tertiary Volcanic Province. Earth Planet. Sci. Lett. 1980, 50, 11-30. [CrossRef]

58. Cabanis, B.; Lecolle, M. Le diagramme La/10-Y/15- $\mathrm{Nb} / 8$ : Un outil pour la discrimination des séries volcaniques et la mise en évidence des procésses de mélange et/ou de contamination crustale. C. R. Acad. Sci. 1989, 2, 2023-2029.

59. McDonough, W.; Sun, S.-S. The composition of the Earth. Chem. Geol. 1995, 120, 223-253. [CrossRef]

60. Le Maitre, R.W.; Bateman, P.; Dudek, A.; Keller, J.; Lameyre, J.; Le Bas, M.J.; Sabine, P.A.; Schmid, R.; Sorensen, H.; Streckeisen, A.; et al. A Classification of Igneou Rocks and Glossary of Terms: Recommendations of the International Union of Geological Sciences Subcommision on the Systematics of Igneous Rocks; Blackwell Science Publishing: Cambridge, UK, 1989; p. 193.

61. Vozár, J.; Spišiak, J.; Vozárová, A.; Bazarnik, J.; Krái, J. Geochemistry and Sr, Nd isotopic composition of the Hronic Upper Paleozoic basic rocks (Western Carpathians, Slovakia). Geol. Carpathica 2015, 66, 3-17. [CrossRef]

62. McDonough, W.; Sun, S.-S.; Ringwood, A.; Jagoutz, E.; Hofmann, A. Potassium, rubidium, and cesium in the Earth and Moon and the evolution of the mantle of the Earth. Geochim. Et Cosmochim. Acta 1992, 56, 1001-1012. [CrossRef]

63. Zindler, A.; Hart, S.R. Chemical geodynamics. Ann. Rev. Earth Planet. Sci. 1986, 14, 493-571. [CrossRef]

64. Hart, S.R.; Hauri, E.H.; Oschmann, L.A.; Whitehead, J.A.; Hart, S.R.; Hauri, E.H.; Oschmann, L.A.; Whitehead, J.A. Mantle Plumes and Entrainment: Isotopic Evidence. Science 1992, 256, 517-520. [CrossRef] [PubMed]

65. Willbold, M.; Stracke, A. Formation of enriched mantle components by recycling of upper and lower continental crust. Chem. Geol. 2010, 276, 188-197. [CrossRef]

66. Kimura, J.; Gill, J.B.; Skora, S.; Van Keken, P.E.; Kawabata, H. Origin of geochemical mantle components: Role of subduction filter. Geochem. Geophys. Geosystems 2016, 17, 3289-3325. [CrossRef]

67. Faure, G. Principles of Isotope Geology, 2nd ed.; John Wiley \& Sons: New York, NY, USA, 1986; p. 589.

68. Romer, R.L.; Hahne, K. Life of the Rheic Ocean: Scrolling through the shale record. Gondwana Res. 2010, 17, 236-253. [CrossRef]

69. Zartman, R.; Doe, B. Plumbotectonics-The model. Tectonophysics 1981, 75, 135-162. [CrossRef]

70. Sommer, C.A.; Barreto, C.J.S.; Lafon, J.M.; Lima, E.F.D.; Alexandre, F.M.; Chemale, F.; Koester, E. Pb isotope geochemistry and reappraisal of Sr-Nd isotopes of the Cerro Morado basic magmatism (Ischigualasto-Villa Union Triassic basin, NW Argentina): Implications for the mantle sources. Braz. J. Geol. 2018, 48, 115-126. [CrossRef]

71. Zou, H. Quantitative Geochemistry; Imperial College Press: London, UK, 2007; p. 287.

72. Hofmann, A.W. Chemical differentiation of the Earth: The relationship between mantle, continental crust, and oceanic crust. Earth Planet. Sci. Lett. 1988, 90, 297-314. [CrossRef]

73. Ito, E.; White, W.M.; Göpel, C. The O, Sr, Nd and Pb isotope geochemistry of MORB. Chem. Geol. 1987, 62, 157-176. [CrossRef]

74. Taylor, S.R.; McLennan, S. The geochemical evolution of the continental crust. Rev. Geophys. 1995, 33, 241-265. [CrossRef]

75. Linnemann, U.; Romer, R.L. The Cadomian Orogeny in Saxo-Thuringia, Germany: Geochemical and Nd-Sr-Pb isotopic characterization of marginal basins with constraints to geotectonic setting and provenance. Tectonophysics 2002, 352, 33-64. [CrossRef]

76. Abdelfadil, K.; Romer, R.; Seifert, T.; Lobst, R. Calc-alkaline lamprophyres from Lusatia (Germany)—Evidence for a repeatedly enriched mantle source. Chem. Geol. 2013, 353, 230-245. [CrossRef] 
77. Hawkesworth, C.J.; Mantovani, M.S.M.; Taylor, P.N.; Palacz, Z. Evidence from the Parana of south Brazil for a continental contribution to Dupal basalts. Nat. Cell Biol. 1986, 322, 356-359. [CrossRef]

78. Marques, L.S.; Dupré, B.; Piccirillo, E. Mantle source compositions of the Paraná Magmatic Province (southern Brazil): Evidence from trace element and $\mathrm{Sr}-\mathrm{Nd}-\mathrm{Pb}$ isotope geochemistry. J. Geodyn. 1999, 28, 439-458. [CrossRef]

79. Lassiter, J.C.; DePaolo, D.J. Plume/Lithosphere Interaction in the Generation of Continental and Oceanic Flood Basalts: Chemical and Isotopic Constraints. In Large Igneous Provinces; American Geophysical Union (AGU): Washington, DC, USA, 2013; Volume 100, pp. 335-355.

80. Vozárová, A.; Túnyi, I. Evidence of the Illawarra Reversal in the Permian sequence of the Hronic Nappe (Western Carpathians, Slovakia). Geol. Carpath. 2003, 54, 229-236.

81. Deroin, J.P.; Bonin, B. Late Variscan tectonomagmatic activity in Western Europe and surrounding areas: The mid-Permian Episode. Boll. Soc. Geol. It. 2003, 2, 169-184. 